\title{
GENOTYPIC VARIATION IN THE RESPONSE OF SORGHUM TO INTERCROPPING WITH COWPEA, AND IN THE EFFECT ON THE ASSOCIATED LEGUME
}

\author{
N.W. GALWEY, M.A. DE QUEIROZ and R.W. WILLEY \\ Department of Applied Biology, University of Cambridge, Pembroke Street, Cambridge, \\ CB2 3DX (Great Britain)
}

(Accepted 24 March 1986)

\section{ABSTRACT}

Galwey, N.W., De Queiroz, M.A. and Willey, R.W., 1986. Genotypic variation in the response of sorghum to intercropping with cowpea, and in the effect on the associated legume. Field Crops Res., 14: 263-290.

Selection of sorghum genotypes for the sorghum-cowpea intercrop system would be simplified if it could be done in sole crop. In order to compare evaluation in sole crop and in the presence of the standard cowpea cultivar C 152, sorghum inbred lines, $F_{1}$ hybrids and land races which differed in maturity date, height and canopy characters were grown in the two systems in two seasons at Hyderabad, India. Cowpea sole crop was included as an additional treatment. Sorghum canopy characters and yield components in intercrop were highly correlated with the same characters in sole crop. However, multiple regression of sorghum grain yield in intercrop on characters measured in intercrop explained more variation than regression on characters measured in sole crop. Characters related to light interception were the most influential in determining sorghum yield, but some genetically determined variation in yield was unexplained by either multiple regression. Characters related to light interception had a negative influence on cowpea yield, though again some variation due to sorghum genotype was unexplained. Thus although the influence of sorghum plant characters on each component crop is predictable, compentsation between the components makes the overall outcome more difficult to predict, and dependent upon which component is favoured by the environment. The sorghum genotypes selected will therefore represent a compromise: they should not be dwarf types, but should be early maturing to escape drought, and have narrow canopies so as not to be too competitive on the cowpea. The final selection should be made in intercrop.

\section{INTRODUCTION}

The association of a low-growing grain legume with a tall cereal is common in tropical agriculture, and there is evidence that such intercrop systems produce higher yields (Krantz et al., 1976, early cereal-pigeon pea; IRRI, 1974, maize-groundnut; Willey and Osiru, 1972, maize-bean; Osiru and Willey, 1972, sorghum-bean) and more stable yields (Papadakis, 1941,

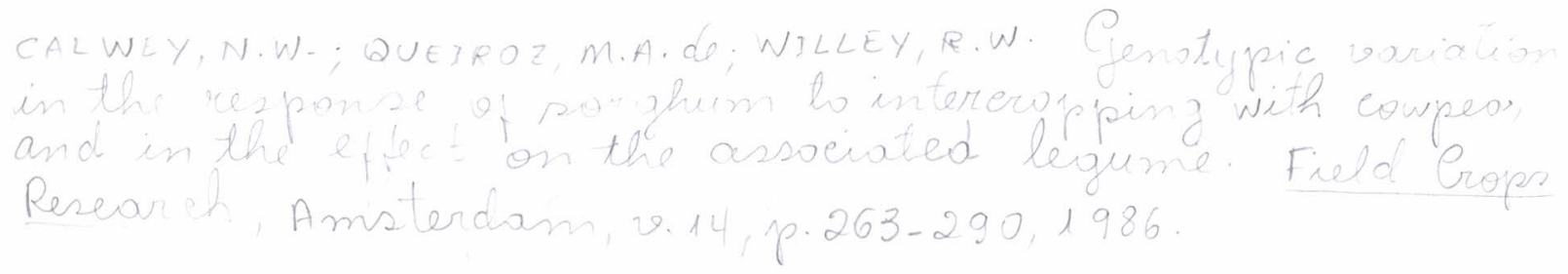


cereal-legume; Fisher, 1976, maize-bean; Rao and Willey, 1980, sorghumpigeon pea) than the corresponding sole crops. Sorghum and cowpea are often intercropped in Africa (Arnon, 1972) and occasionally in India (Aiyer, 1949). In the present study the major interest in this association is its potential for north-eastern Brazil where, because of the semi-arid conditions, there is a strong case for introducing sorghum to replace some of the maize that is so commonly grown in association with cowpea. However, plant breeders have not concentrated on producing improved genotypes for this system, mainly because it is used in subsistence agriculture, and this sector of the economy cannot finance investment in breeding. Breeders are further deterred because the simultaneous improvement of two crops has sometimes been seen as dauntingly complicated (Hamblin et al., 1976), whereas by other workers it is assumed that selection for sole crop will produce the genotypes best adapted for intercrop. Between these extreme points of view lies the observation that the taller sorghum plant must largely determine the performance of the cowpea, and hence of the intercrop as a whole. In this case it should be possible to select sorghum genotypes in the presence of a standard cowpea genotype, but the selections will not necessarily be the same as those for sole crop.

Most studies of intercrops have concentrated on agronomic aspects, but some have provided information relevant to this last approach, the selection of genotypes. Baker (1974) found similar ranking of yields of four cultivars of sorghum in monoculture, and when intercropped with a shorter millet (Pennisetum typhoides L.). Finlay (1974), working with twelve soybean cultivars in monoculture, and intercropped with cereals (maize, sorghum and millet) found that the cultivar $X$ system interaction was not significant. When bush bean (Phaseolus vulgaris L.) cultivars with diverse growth habits were grown in monoculture, and intercropped with a tall double-cross maize hybrid, there were significant correlations between bean yields in the two cropping systems (Francis et al., 1978a), but when climbing varieties of $P$. vulgaris $L$. were used there were no such significant correlations (Francis et al:, 1978b). This may indicate that a standard legume genotype can only be used when the legume component is lowgrowing. Green et al. (1981) selected pigeon pea lines in sole crop, and intercropped with sorghum, during 4 years, and found that $29 \%, 67 \%, 75 \%$, and $0 \%$ of the selected lines were common to the two systems. In the cases where the correlation between yield in the two cropping systems was poor, it is likely that the genotypes had morphological or physiological characteristics which adapted them specifically to one system or the other.

The objective of the present study was to determine how the grain and fodder yield of both sorghum and cowpea, and the overall value of intercrop as indicated by the total land equivalent ratio (LER), were related to other characteristics of the sorghum plants, measured either in intercrop or in sole crop. This would indicate whether it is necessary to select genotypes specifically for use in intercrop, and if so whether such genotypes can 
be identified in sole crop thereby eliminating the need for separate trials.

Competition between sorghum and cowpea takes place as soon as some environmental resources become in short supply for at least one of the intercrop species (Clements et al., 1929), well before final height of the cereal is reached. Therefore in addition to the final height, the time and rate at which this height was achieved were measured. The width of the canopy above the cowpea, as well as its height, affects the efficiency of sorghum genotypes in intercepting and using light themselves and largely determines the amount of light that reaches the associated legume. However, this character has apparently not been measured before in a range of cereal genotypes in intercropping. The final canopy width, and the time and rate of achieving this, were therefore measured.

\section{MATERIALS AND FIELD METHODS}

\section{Location and environment}

The experiments were carried out in the 1981 and 1982 growing seasons at the International Crops Research Institute for the Semi-Arid Tropics (ICRISAT), about $25 \mathrm{~km}$ north of Hyderabad, India, between 530 and $560 \mathrm{~m}$ above sea level. The soil is classified as medium deep black (50$90 \mathrm{~cm}$ depth) (Singh and Krantz, 1976), a clay loam with good moisture holding capacity, but low in available nutrients. In 1981 the total rainfall was $1070.7 \mathrm{~mm}, 34 \%$ above the normal value. There was no moisture stress during crop growth but there were prolonged periods when the soil was saturated. However, in the 1982 rainy season the total rainfall was 649.3 $\mathrm{mm}, 19 \%$ below normal, and some dry spells occurred, subjecting the crop to moisture stress.

\section{Choice of genotypes}

The 23 sorghtum genotypes grown in 1981 formed three groups, namely 18 inbred lines from different stages of yield improvement, 3 commercial hybrids, and 2 local types (land races), with wide genetic variation between and within groups. The details of each group are as follows.

The inbred lines formed two sub-groups. One comprised 12 sister lines from the crosses $555 \times 148$ and $555 \times 168$, the lines 148 and 168 themselved being derived from the same cross. The parents are improved sorghum lines selected for grain yield in the All India Coordinated Sorghum Improvement Programme (AICSIP). The sister lines were selected as ICRISAT for contrasting height and maturity, with the intention of avoiding the confounding effects of diverse genetic background. They do not differ very much in other canopy features such as leaf arrangement and their variability was therefore supplemented with other genotypes. The second sub-group comprised three improved lines developed by AICSIP, namely 
148 (a parent of the sister lines), CS 3541 and 2219B, and three improved lines developed at ICRISAT, namely SPV 351, S902, and M66433.

The three hybrids, CSH 5, CSH 6, and CSH 9, also developed by AICSIP have the same male parent CS 3541 , but different female parents, $2077 \mathrm{~A}$, $2219 \mathrm{~A}$ and $296 \mathrm{~A}$, respectively. CSH 6 , released in 1976, is the highest yielding and most popular hybrid in India, especially in the reliable rainfall areas of Maharastra State. CSH 5 and $\mathrm{CSH} 9$ are good yielders of grain and fodder, a little later maturing than $\mathrm{CSH} 6$, and quite popular in the most important sorghum-growing areas.

The two land races were E 35-1 from Ethiopia, tall and late, and IS 9742 from Sudan, tall and early. They were selected by farmers in areas where intercropping is common and have good grain quality and fodder yield, but low grain yield/unit area. Such cultivars evolved at low population densities and at high densities the grain yield decreases drastically, sometimes reaching zero (Stoop, 1981).

These 23 genotypes included three dwarf types, namely S1021, 2219B and M66433.

In 1982 a subset of the sorghum genotypes used in 1981 was grown, keeping the same range of variability but omitting some genotypes which had similar features.

The cowpea variety C 152 , which was selected at the Indian Agricultural Research Institute, has a semi-erect growth habit and flowers 45 days from sowing.

\section{Experimental design}

All plots were sown with a seeder on $45 \mathrm{~cm}$ rows. Sorghum was thinned to a within-row spacing of $12 \mathrm{~cm}$, to try to achieve the recommended density of 18 plants $/ \mathrm{m}^{2}$ for the sole crop. Cowpea was unthinned and the seed rate was intended to give about the recommended density of 30 plants $/ \mathrm{m}^{2}$ for the sole crop. The intercrop plots were sown in an arrangement of 1 row sorghum to 2 rows cowpea, giving plant densities in a replacement series (de Wit, 1960) of $1 / 3$ sorghum and $2 / 3$ cowpea.

A split plot design was used, with three replications, in which the sorghum genotypes were allocated to main plots and the two systems (intercrop and sole crop) to sub-plots. The sole crop cowpea occupied an additional main plot. The main plots were arranged in randomised complete blocks. In 1981 the sub-plots were $3.6 \mathrm{~m} \times 10 \mathrm{~m}$ for sole crop and 4.5 $\times 10 \mathrm{~m}$ for intercrop, but in 1982 the length of all sub-plots was decreased to $9 \mathrm{~m}$. The central $7 \mathrm{~m}$ of the four central rows of the sole crop plots were harvested in 1981 giving an area of $12.6 \mathrm{~m}^{2}$ per plot. In 1982 the equivalent area was $10.8 \mathrm{~m}^{2}$. In the intercrop plots the two central sorghum rows plus the two central cowpea rows were harvested, and yields per unit area were then adjusted to the correct 1 sorghum: 2 cowpea proportions. 


\section{Agronomic management}

Preceding the experiment the field was sown with a uniform cover crop of fodder maize. In $198118 \mathrm{~kg} /$ ha of $\mathrm{N}$ and $46 \mathrm{~kg} / \mathrm{ha}$ of $\mathrm{P}_{2} \mathrm{O}_{5}$ was applied basally, and $62 \mathrm{~kg} / \mathrm{ha}$ of $\mathrm{N}$ was applied as a top dressing to the sorghum crop, divided between applications at 30 and 60 days after sorghum emergence. In $1982,60 \mathrm{~kg} / \mathrm{ha}$ of $\mathrm{P}_{2} \mathrm{O}_{5}$ was applied basally and $80 \mathrm{~kg} / \mathrm{ha}$ of $\mathrm{N}$ was topdressed to the sorghum crop in a single application three weeks after emergence. Six insecticidal applications were made to the sorghum in each experiment, providing intensive protection, and a single insecticide application was made to the cowpea. The experiments were weeded by hand when necessary.

\section{Measurements}

The following aspects of sorghum were recorded:

(i) plants $/ \mathrm{m}^{2}$ at harvest, estimated from counts of a $1 \mathrm{~m}$ section of each harvested row in each sub-plot.

(ii) maximum plant height attained (Y), from the ground to the highest part the part of the flag leaf, measured on eight adjacent plants in each sub-plot in both cropping systems. The time $(X$, in days from emergence) and the rate $(\mathrm{Y} / \mathrm{X})$ of achieving maximum height were also estimated for each genotype in the intercrop plots, averaged over replications.

(iii) maximum plant canopy width, time of achieving maximum width, and rate of achieving maximum width, measured on the same basis as the corresponding variables for plant height.

(iv) days from emergence to $50 \%$ flowering, averaged over replications and cropping systems.

(v) leaf area of eight adjacent plants in each sub-plot.

(vi) the percentage of transmission of photosynthetically active radiation through the sorghum canopy to the top of the cowpea recorded on the day (or following day) on which plant height and canopy width were measured at three points along the intercrop sub-plot, using a T-meter apparatus (Williams and Austin, 1977) modified at ICRISAT.

(vii) dry fodder and grain yield $/ \mathrm{m}^{2}$ and the yield components head number/ plant, grains/head and weight/grain.

From the cowpea plants the dry fodder and grain yield $/ \mathrm{m}^{2}$ and the plants/ $\mathrm{m}^{2}$, pods/plant, grains/pod and weight/grain were recorded.

\section{STATISTICAL METHODS}

\section{Individual characters}

Analysis of variance within each cropping system was performed on the variables measured in each sub-plot, and the genotypic variation was 
partitioned into between- and within-groups components. For days to flowering, time to maximum canopy height and width and rate of achieving maximum height and width, only average values over replications were available and hence this analysis of variance was not possible.

The way in which the response to intercropping of a particular genotype differs from the general pattern of response can be measured by three methods, which have been compared by Galwey and Evans (1982) in the context of genotype evaluation with and without insecticidal protection. These measurements are:

(i) the mean deviation, for each genotype, from a regression of genotype means in intercrop on genotype means in sole crop.

(ii) the genotype $X$ cropping system interaction effect, for each genotype, from a split-plot analysis of variance. This method is valid only when the slope of the regression line from method (i) is near 1 , though it has been widely used in intercropping studies (e.g. Francis et al., 1978a).

(iii) the mean ratio, for each genotype, of the value in intercropping to the value in sole cropping, or the mean log (ratio) if this gives a more homogenous and more nearly normal distribution of the residual values. This is valid only if the intercept of the regression line from method (i) is close to 0 , a condition which was tested and found to be met for all variables except dry fodder yield and heads/plant. This method was therefore used.

\section{Sorghum grain yield related to sorghum plant characters}

If the genotypes respond differently to intercropping in terms of their yield, it is of relevance to determine whether this response can be predicted from other sorghum plant characters, and particularly from measurements in sole crop.

Simple regression analyses of the mean sorghum intercrop yield of each genotype on sorghum characters in sole crop were performed, and a multiple regression model was constructed by the stepwise method (Draper and Smith, 1981) using the critical value $t=1.5$ for the inclusion of a term in the model. After the establishment of the final model, a model involving the same variables was fitted treating each plot as a separate observation, and the residual variation in sorghum intercrop yield was subjected to analysis of variance in order to determine whether some genotypic variation was still unaccounted for. Such unaccounted-for variation would imply that some variable under genetic control, important for explaining sorghum yield in intercropping, had not been measured.

Similar simple and multiple regressions and analyses of variance were carried out with variables measured in intercrop replacing those measured in sole crop, to determine whether this changed the form or closeness of the relationships. The variables included those measured only in intercrop, which cannot be measured at sole crop density. 
Like sorghum intercrop yield, cowpea intercrop yield was related to sorghum characters in sole crop and intercrop by simple and multiple regression, and analysis of variance was used to detect additional effects of sorghum genotype. Cowpea LER was not analysed, since there was only one cowpea sole crop treatment and hence variation in LER would follow that in intercrop yield.

Lastly the sorghum, cowpea and total LERs were correlated with the sorghum characters found to be important in the preceding analyses, and with each other.

\section{RESULTS AND DISCUSSION}

\section{Individual characters}

The analyses of variance of sorghum characters measured within each cropping system are presented in Table 1 . The following conventions for statistical significance are used throughout: $n s=$ not significant; $*=P$ $<0.05 ; * *=P<0.01 ; * * *=P<0.001$. In Table 1 , the variance is partitioned between sister lines, improved lines, hybrids and land races, while in Table 2 the alternative partitioning between dwarf and non-dwarf groups of genotypes is used. In Table 3 the minimum, mean and maximum values and the coefficient of variation of each character are presented. The partitioning into dwarf and non-dwarf groups was naturally more successful in explaining variation in canopy height, but also produced higher betweengroups $F$ ratios for total leaf area and dry fodder yield, though not grain yield and its components, except wt/grain in sole crop in 1982. The coefficient of variation was as low as $5.3 \%$ for canopy height, the most stable character measured, but $27.4 \%$ for intercrop grain yield in 1981. Coefficients of variation were generally lower in 1982, except for leaf area. There was significant variation between sorghum genotypes in the number of plants $/ \mathrm{m}^{2}$ with values ranging from 91 to $115 \%$ of the intended values in 1981 and from 98 to $126 \%$ of the intended values in 1982 . This unwanted variation may have had some effect on the other characters measured. The correlation between the number of plants $/ \mathrm{m}^{2}$ in sole and intercrop in 1982 was $0.719, P<0.01$, indicating that generally the same genotypes suffered from poor establishment in each system. The correlation between the number of plants $/ \mathrm{m}^{2}$ in sole crop in 1981 and 1982 was -0.514 , $\mathrm{ns}$, indicating that different genotypes suffered from poor establishment in the two years. It is not possible to adjust for the effect of plant population using covariance analysis, since population varied between genotypes and any adjustment might eleminate important variation due to other causes. The correlations between plant population and other variables were not consistent: for example the correlation between plants $/ \mathrm{m}^{2}$ and sorghum grain yield in sole crop was 0.413 , almost significant, in 1981 
Analysis of variance of sorghum plant characters within each cropping system with the partition of genotype effects among groups bred by different methods

\begin{tabular}{|c|c|c|c|c|c|c|c|c|c|c|c|c|c|c|c|c|c|c|}
\hline \multirow[b]{3}{*}{ Year } & \multirow{2}{*}{\multicolumn{2}{|c|}{$\begin{array}{l}\text { Character } \\
\text { Cropping system }\end{array}$}} & \multirow{2}{*}{\multicolumn{2}{|c|}{$\begin{array}{l}\text { Plants } / \mathrm{m}^{2} \\
\text { Sole }\end{array}$}} & \multirow{2}{*}{\multicolumn{2}{|c|}{ Inter }} & \multirow{2}{*}{\multicolumn{4}{|c|}{$\begin{array}{l}\text { Canopy height } \\
\text { Sole Inter }\end{array}$}} & \multirow{2}{*}{\multicolumn{4}{|c|}{$\begin{array}{l}\text { Total leaf area } \\
\text { Sole Inter }\end{array}$}} & \multirow{2}{*}{\multicolumn{4}{|c|}{$\begin{array}{l}\text { Dry fodder yield } \\
\text { Sole }\end{array}$}} \\
\hline & & & & & & & & & & & & & & & & & Inter & \\
\hline & $\begin{array}{l}\text { Source of } \\
\text { variation }\end{array}$ & DF & $F$ & $P$ & $F$ & $P$ & $F$ & $P$ & $F$ & $P$ & $F$ & $P$ & $F$ & $P$ & $F$ & $P$ & $F$ & $P$ \\
\hline \multirow[t]{7}{*}{1981} & Genotypes & 22 & 1.83 & $*$ & 1.13 & ns & 109.32 & $* * *$ & 102.21 & $* * *$ & 6.18 & $* * *$ & 4.75 & $* * *$ & 11.14 & $* * *$ & 10.99 & *** \\
\hline & Amor & 3 & 1.55 & ns & 1.28 & ns & 332.59 & $* * *$ & 371.42 & $* * *$ & 2.19 & ns & 0.98 & $\mathrm{~ns}$ & 13.68 & $* * *$ & & $* * *$ \\
\hline & Sister & 11 & 2.59 & * & 1.04 & ns & 91.06 & *** & 72.93 & $* * *$ & 3.08 & ** & 2.69 & $* *$ & 7.66 & $* * *$ & 7.95 & *** \\
\hline & Improved lines & 5 & 0.69 & ns & 1.38 & ns & 72.86 & $* * *$ & 59.95 & $* * *$ & 8.04 & $* * *$ & 5.26 & $* * *$ & 20.14 & $* * *$ & 12.95 & $* * *$ \\
\hline & Hybrids & 2 & 1.56 & ns & 0.51 & ns & 6.28 & $* *$ & 8.84 & $* * *$ & 3.34 & $*$ & 5.15 & $* *$ & 0.28 & ns & & ns \\
\hline & Land races & 1 & 1.24 & ns & 1.56 & ns & 6.61 & $*$ & 14.81 & $* * *$ & 48.65 & $* * *$ & 35.44 & $* * *$ & 18.58 & $* * *$ & 10.62 & $* *$ \\
\hline & Error & 44 & & & & & & & & & & & & & & & & \\
\hline \multirow[t]{7}{*}{1982} & Genotypes & 13 & $\begin{array}{r}3.89 \\
10.73\end{array}$ & & $\begin{array}{l}4.24 \\
9.04\end{array}$ & **** & 33 & & $88 . C$ & & 62 & * & 4.59 & ${ }^{* * *}$ & & *** & & \\
\hline & $\begin{array}{l}\text { Amo } \\
\text { Siste }\end{array}$ & $\begin{array}{l}3 \\
3\end{array}$ & $\begin{array}{r}10.73 \\
0.10\end{array}$ & ns & $\begin{array}{l}9.04 \\
3.87\end{array}$ & ** & $\begin{array}{l}67.10 \\
22.64\end{array}$ & $\begin{array}{l}* * * \\
* * *\end{array}$ & $\begin{array}{r}110.69 \\
26.68\end{array}$ & $*$ & $\begin{array}{l}6.35 \\
0.45\end{array}$ & $\begin{array}{l}* * \\
\mathrm{~ns}\end{array}$ & $\begin{array}{r}11.81 \\
0.96\end{array}$ & $\begin{array}{l}* * \\
\mathrm{~ns}\end{array}$ & $\begin{array}{l}10 \\
96\end{array}$ & $\begin{array}{l}* * * \\
* * *\end{array}$ & $\begin{array}{l}57.81 \\
18.49\end{array}$ & $\begin{array}{l}* * * \\
* * *\end{array}$ \\
\hline & d lines & 4 & 0.60 & ns & 1.45 & ns & 19.70 & $* * *$ & 33. & ** & 1.02 & ns & 1.96 & ns & & $* * *$ & 1.75 & $* * *$ \\
\hline & $\mathrm{Hyb}$ & 2 & 6.99 & $* *$ & 0.97 & ns & & ns & 4.50 & $*$ & 2.60 & ns & 6.00 & ** & & $* *$ & & $* * *$ \\
\hline & Land $x$ & 1 & 1.66 & ns & 8.68 & $* *$ & 24.10 & ${ }^{* * * *}$ & 58.56 & $* * *$ & 4.46 & $*$ & 1.53 & ns & 38.46 & $* * *$ & 81.29 & $* * *$ \\
\hline & Error & 26 & & & & & & & & & & & & & & & & \\
\hline & \multicolumn{2}{|l|}{$\begin{array}{l}\text { Character } \\
\text { Cropping system }\end{array}$} & \multicolumn{2}{|c|}{$\begin{array}{l}\text { Grain yield } \\
\text { Sole }\end{array}$} & Inter & & \multicolumn{2}{|c|}{$\begin{array}{l}\text { Heads/plant } \\
\text { Sole }\end{array}$} & Inter & & \multicolumn{2}{|c|}{$\begin{array}{l}\text { Grains/head } \\
\text { Sole }\end{array}$} & \multicolumn{2}{|l|}{ Intex } & \multicolumn{2}{|c|}{$\begin{array}{l}\text { Weight/grain } \\
\text { Sole }\end{array}$} & Inter & \\
\hline Year & $\begin{array}{l}\text { Source of } \\
\text { variation }\end{array}$ & $\mathrm{DF}$ & $F$ & $P$ & $F$ & $P$ & $F$ & $P$ & $F$ & $P$ & $F$ & $P$ & $F$ & $P$ & $F$ & $P$ & $F$ & $P$ \\
\hline \multirow[t]{7}{*}{1981} & Genotypes & 22 & 15.28 & $* * *$ & 8.75 & $* * *$ & 3.75 & $* * *$ & 0.91 & ns & 15.18 & $* * *$ & 9.12 & **** & 13. & $* * *$ & 11.00 & $* * *$ \\
\hline & Ams & 3 & 72.57 & $* * *$ & 41.78 & ** & & $*$ & & & & ** & 45. & & & & & \\
\hline & Siste & 11 & 4.37 & $* * *$ & 2.53 & $*$ & 1.82 & ns & 85 & & 33 & $* * *$ & 3.58 & $* *$ & 8. & $* * *$ & 6.67 & $* * *$ \\
\hline & $\operatorname{Imp}$ & 5 & & $* * *$ & 4.97 & ** & & ns & & & & $* * *$ & & $* *$ & & $* *$ & 1.84 & ns \\
\hline & $\mathrm{Hybr}$ & 2 & 11.68 & $* * *$ & & ns & & ns & & ns & 0.93 & ns & .47 & ns & 6. & $* *$ & 2.80 & $\mathrm{~ns}$ \\
\hline & Land $\mathrm{r}$ & 1 & 12.58 & $* * *$ & 10.30 & ** & 39.33 & $* * *$ & 0.10 & $\mathrm{~ns}$ & 1.68 & ns & 0.73 & ns & 114.06 & $* * *$ & 97.60 & $* * *$ \\
\hline & Error & 44 & & & & & & & & & & & & & & & & \\
\hline \multirow[t]{7}{*}{1982} & Genoty & 13 & 1 & $* * *$ & 11.9 & $* * *$ & & ns & & & & $* * *$ & & & & & & \\
\hline & & 3 & 38. & $* * *$ & 44.8 & $* * *$ & & ns & 34. & & & $* * *$ & & $* * *$ & & & & \\
\hline & & 3 & & ns & & ns & & ns & & s & & $* * *$ & & $* *$ & & $* * *$ & & $* * *$ \\
\hline & Im & 4 & & ns & & ns & & ns & & & & & & $* *$ & 29.20 & $* * *$ & 11.66 & $* * *$ \\
\hline & & 2 & 7.12 & $* *$ & 5.24 & $*$ & 0.04 & $\mathrm{~ns}$ & 0.47 & ns & 10.60 & $* * *$ & 8.71 & $* *$ & 1.22 & ns & 2.45 & ns \\
\hline & Lan & 1 & 0.56 & ns & 0.04 & ns & 18.85 & $* * *$ & 156.26 & $* * *$ & 33.60 & $* * *$ & 70.09 & $* * *$ & 116.25 & $* * *$ & 224.00 & $* * *$ \\
\hline & Error & 26 & & & & & & & & & & & & & & & & \\
\hline
\end{tabular}


TABLE 2

Analysis of variance of sorghum plant characters within each cropping system with the partition of genotype effects among dwarf and non-dwarf types

\begin{tabular}{|c|c|c|c|c|c|c|c|c|c|c|c|c|c|c|c|c|c|c|}
\hline \multirow[b]{3}{*}{ Year } & \multirow{2}{*}{\multicolumn{2}{|c|}{$\begin{array}{l}\text { Character } \\
\text { Cropping system }\end{array}$}} & \multirow{2}{*}{\multicolumn{2}{|c|}{$\begin{array}{l}\text { Plants } / \mathrm{m}^{2} \\
\text { Sole }\end{array}$}} & \multirow{2}{*}{\multicolumn{2}{|c|}{ Inter }} & \multirow{2}{*}{\multicolumn{4}{|c|}{$\begin{array}{l}\text { Canopy height } \\
\text { Sole Int }\end{array}$}} & \multirow{2}{*}{\multicolumn{4}{|c|}{$\begin{array}{l}\text { Total leaf area } \\
\text { Sole In }\end{array}$}} & \multicolumn{4}{|c|}{ Dry fodder yield } \\
\hline & & & & & & & & & & & & & & & & & Inter & \\
\hline & $\begin{array}{l}\text { Source of } \\
\text { variation }\end{array}$ & DF & $F$ & $P$ & $F$ & $P$ & $F$ & $P$ & $F$ & $P$ & $F$ & $P$ & $F$ & $P$ & $F$ & $P$ & $F$ & $P$ \\
\hline \multirow[t]{3}{*}{1981} & Among groups & 1 & 3.21 & ns & 4.38 & $*$ & 766.37 & $* * *$ & 742.96 & $* * *$ & 50.16 & $* * *$ & 17.59 & $* * *$ & 117.76 & $* * *$ & 106.49 & $* * *$ \\
\hline & Dwarf ty & 2 & 0.73 & ns & 0.27 & ns & 0.40 & ns & 0.41 & $\mathrm{~ns}$ & 0.70 & ns & 1.90 & ns & 0.55 & ns & 0.72 & ns \\
\hline & Non-dwarf types & $\begin{array}{l}19 \\
44\end{array}$ & 1.87 & ns & 1.05 & ns & 85.04 & $* * *$ & 79.21 & $* * *$ & 4.45 & $* * *$ & 4.38 & $* * *$ & 6.64 & $* * *$ & 7.05 & $* * *$ \\
\hline \multirow[t]{4}{*}{1982} & Among groups & 1 & 5.65 & * & 0.21 & ns & 149.08 & $* * *$ & 259.57 & $* * *$ & 7.85 & ** & 17.80 & $* * *$ & 98.01 & $* * *$ & 152.38 & $* * *$ \\
\hline & Dwarf types & 2 & 2.61 & $*$ & 7.02 & $* *$ & 0.17 & ns & 0.60 & ns & 1.20 & ns & 2.20 & ns & 6.44 & $* *$ & 4.86 & $*$ \\
\hline & Non-dwarf types & $\begin{array}{l}10 \\
26\end{array}$ & 3.96 & $* *$ & 4.09 & $* *$ & 22.76 & $* * *$ & 35.35 & $* * *$ & 2.39 & $*$ & 3.75 & $* *$ & 17.47 & $* * *$ & 24.60 & $* * *$ \\
\hline & \multicolumn{2}{|l|}{$\begin{array}{l}\text { Character } \\
\text { Cropping system }\end{array}$} & \multicolumn{2}{|c|}{$\begin{array}{l}\text { Grain yield } \\
\text { Sole }\end{array}$} & Inter & & \multicolumn{2}{|c|}{$\begin{array}{l}\text { Heads/plant } \\
\text { Sole }\end{array}$} & \multicolumn{2}{|l|}{ Inter } & \multicolumn{2}{|c|}{$\begin{array}{l}\text { Grains/head } \\
\text { Sole }\end{array}$} & Inter & & \multicolumn{2}{|c|}{$\begin{array}{l}\text { Weight/grain } \\
\text { Sole }\end{array}$} & Inter & \\
\hline Year & $\begin{array}{l}\text { Source of } \\
\text { variation }\end{array}$ & $\mathrm{DF}$ & $F$ & $P$ & $F$ & $P$ & $F$ & $P$ & $F$ & $P$ & $F$ & $P$ & $F$ & $P$ & $F$ & $P$ & $F$ & $P$ \\
\hline \multirow[t]{3}{*}{1981} & Among groups & 1 & 4.43 & $*$ & 8.63 & $* *$ & 1.33 & ns & 0.99 & ns & 6.72 & * & 5.47 & * & 6.58 & * & 4,33 & $*$ \\
\hline & Dwarf ty & 2 & 11.51 & $* * *$ & 3.10 & ns & 9.59 & $* * *$ & 4.63 & $*$ & 7.56 & $* *$ & 2.06 & ns & 3.79 & $*$ & 1.00 & $\mathrm{~ns}$ \\
\hline & Non-dwarf types & $\begin{array}{l}19 \\
44\end{array}$ & 16.24 & $* * *$ & 9.36 & $* * *$ & 3.26 & $* * *$ & 0.51 & ns & 16.48 & $* * *$ & 10.05 & $* * *$ & 14.38 & $* * *$ & 12.40 & $* * *$ \\
\hline \multirow[t]{3}{*}{1982} & Among groups & 1 & 3.29 & $*$ & 3.39 & ns & 0.41 & ns & 0.28 & ns & 43.96 & $* * *$ & 0.33 & $\mathrm{~ns}$ & 122.01 & $* * *$ & 34.20 & $* * *$ \\
\hline & Dwarf types & 2 & 1.69 & ns & 0.63 & ns & 1.17 & ns & 2.01 & ns & 3.75 & * & 0.05 & ns & 3.56 & ns & 0.04 & ns \\
\hline & Non-dwars types & 10 & 14.06 & $* * *$ & 15.05 & $* * *$ & 2.14 & ns & 26.81 & $* * *$ & 55.06 & $* * *$ & 65.15 & $* * *$ & 51.90 & $* * *$ & 44.87 & *** \\
\hline
\end{tabular}


TABLE 3

Minimum, mean and maximum genotype means, and coefficients of variation, of sorghum plant characters

\begin{tabular}{|c|c|c|c|c|c|c|c|c|c|}
\hline \multirow[t]{3}{*}{ Charactex } & \multirow{3}{*}{$\begin{array}{l}\text { Crop- } \\
\text { ping } \\
\text { sys- } \\
\text { tem }\end{array}$} & \multicolumn{8}{|l|}{ Year } \\
\hline & & \multicolumn{4}{|l|}{1981} & \multicolumn{4}{|l|}{1982} \\
\hline & & Min. & Mean & Max. & $\begin{array}{l}\text { C.V. } \\
(\%)\end{array}$ & Min. & Mean & Max. & $\begin{array}{l}\text { C.V. } \\
(\%)\end{array}$ \\
\hline Plant $/ \mathrm{m}^{2}$ & $\begin{array}{l}\text { Sole } \\
\text { Inter }\end{array}$ & $\begin{array}{r}17.41 \\
6.10\end{array}$ & $\begin{array}{r}19.26 \\
6.61\end{array}$ & $\begin{array}{r}20.82 \\
7.18\end{array}$ & $\begin{array}{l}5.7 \\
8.2\end{array}$ & $\begin{array}{r}16.85 \\
6.50\end{array}$ & $\begin{array}{r}20.28 \\
7.07\end{array}$ & $\begin{array}{r}21.79 \\
7.84\end{array}$ & $\begin{array}{l}5.2 \\
4.4\end{array}$ \\
\hline Canopy height (cm) & $\begin{array}{l}\text { Sole } \\
\text { Inter }\end{array}$ & $\begin{array}{l}77.3 \\
77.7\end{array}$ & $\begin{array}{l}147.3 \\
146.3\end{array}$ & $\begin{array}{l}239.3 \\
247.7\end{array}$ & $\begin{array}{l}5.3 \\
5.3\end{array}$ & $\begin{array}{l}81.7 \\
76.7\end{array}$ & $\begin{array}{l}122.3 \\
117.4\end{array}$ & $\begin{array}{l}200.0 \\
191.7\end{array}$ & $\begin{array}{l}8.7 \\
6.6\end{array}$ \\
\hline $\begin{array}{l}\text { Total leaf area } \\
\left(\mathrm{cm}^{2} / \text { plant }\right)\end{array}$ & $\begin{array}{l}\text { Sole } \\
\text { Inter }\end{array}$ & $\begin{array}{l}1418 \\
1473\end{array}$ & $\begin{array}{l}2732 \\
2301\end{array}$ & $\begin{array}{l}3915 \\
3222\end{array}$ & $\begin{array}{l}16.1 \\
15.6\end{array}$ & $\begin{array}{l}1515 \\
1708\end{array}$ & $\begin{array}{l}2706 \\
3111\end{array}$ & $\begin{array}{l}4668 \\
4689\end{array}$ & $\begin{array}{l}31.9 \\
24.3\end{array}$ \\
\hline $\begin{array}{l}\text { Dry fodder yield } \\
\left(\mathrm{g} / \mathrm{m}^{2}\right)\end{array}$ & $\begin{array}{l}\text { Sole } \\
\text { Inter }\end{array}$ & $\begin{array}{l}94.6 \\
34.4\end{array}$ & $\begin{array}{r}187.2 \\
96.3\end{array}$ & $\begin{array}{l}290.0 \\
171.6\end{array}$ & $\begin{array}{l}12.9 \\
17.1\end{array}$ & $\begin{array}{r}122.8 \\
57.2\end{array}$ & $\begin{array}{l}254.2 \\
133.5\end{array}$ & $\begin{array}{l}443.9 \\
264.8\end{array}$ & $\begin{array}{l}11.8 \\
11.9\end{array}$ \\
\hline Grain yield $\left(\mathrm{g} / \mathrm{m}^{2}\right)$ & $\begin{array}{l}\text { Sole } \\
\text { Inter }\end{array}$ & $\begin{array}{l}10.4 \\
11.7\end{array}$ & $\begin{array}{l}71.5 \\
48.5\end{array}$ & $\begin{array}{l}172.3 \\
105.1\end{array}$ & $\begin{array}{l}23.3 \\
27.4\end{array}$ & $\begin{array}{l}98.1 \\
59.3\end{array}$ & $\begin{array}{r}156.1 \\
91.2\end{array}$ & $\begin{array}{l}226.1 \\
141.5\end{array}$ & $\begin{array}{l}11.9 \\
12.9\end{array}$ \\
\hline Heads/plant & $\begin{array}{l}\text { Sole } \\
\text { Inter }\end{array}$ & $\begin{array}{l}0.590 \\
0.900\end{array}$ & $\begin{array}{l}0.914 \\
1.011\end{array}$ & $\begin{array}{l}1.163 \\
1.197\end{array}$ & $\begin{array}{l}12.2 \\
11.8\end{array}$ & $\begin{array}{l}0.885 \\
0.989\end{array}$ & $\begin{array}{l}1.029 \\
1.106\end{array}$ & $\begin{array}{l}1.257 \\
1.776\end{array}$ & $\begin{array}{r}10.2 \\
6.8\end{array}$ \\
\hline Grains/head & $\begin{array}{l}\text { Sole } \\
\text { Inter }\end{array}$ & $\begin{array}{l}248 \\
403\end{array}$ & $\begin{array}{r}780 \\
1269\end{array}$ & $\begin{array}{l}1342 \\
2292\end{array}$ & $\begin{array}{l}18.4 \\
23.2\end{array}$ & $\begin{array}{l}49 \\
85\end{array}$ & $\begin{array}{l}1196 \\
1774\end{array}$ & $\begin{array}{l}1891 \\
2872\end{array}$ & $\begin{array}{r}10.6 \\
9.4\end{array}$ \\
\hline Weight/grain (mg) & $\begin{array}{l}\text { Sole } \\
\text { Inter }\end{array}$ & $\begin{array}{l}12.77 \\
13.60\end{array}$ & $\begin{array}{l}18.07 \\
19.79\end{array}$ & $\begin{array}{l}28.73 \\
31.73\end{array}$ & $\begin{array}{l}9.3 \\
9.8\end{array}$ & $\begin{array}{l}16.90 \\
20.51\end{array}$ & $\begin{array}{l}21.68 \\
24.04\end{array}$ & $\begin{array}{l}35.03 \\
39.21\end{array}$ & $\begin{array}{l}5.4 \\
5.6\end{array}$ \\
\hline
\end{tabular}

and $-0.226, \mathrm{~ns}$, in 1982 . It was therefore considered reasonable to proceed on the assumption that the effects of genotypes generally arose from causes other than their plant population.

The genotypes varied substantially for several characters. These included flowering date and time and rate of achievement of maximum canopy height and width, which could not be subjected to analysis of variance but which are highly heritable. For some characters there was substantially more variation between than within groups of genotypes bred by different methods. The improved lines were particularly short, whereas the local types were particularly tall and had wide canopies. The improved lines and hybrids had high grain yields. But many characters varied both within and between groups, and indeed even among genotypes of similar height and width there was considerable variation in leaf area.

The analyses of variance for cowpea plants $/ \mathrm{m}^{2}$, grain and dry fodder yield and yield components are presented in Table 4 . The densities in both seasons and both systems were about $155 \%$ of the intended values, but did not vary significantly between sorghum genotypes. This excess in cowpea population must have affected some yield components but the yield/ha of grain and particularly of dry fodder remains almost the same over a wide range of cowpea population density above 200000 plants/ha (Willey and Heath, 1969). 
TABLE 4

Analysis of variance of cow pea plant characteristics over both cropping systems and in intercrop only

\begin{tabular}{|c|c|c|c|c|c|c|c|c|c|c|c|c|c|c|}
\hline \multirow[b]{2}{*}{ Year } & \multirow{2}{*}{$\begin{array}{l}\text { Characters } \\
\text { Source of } \\
\text { variation }\end{array}$} & \multirow[b]{2}{*}{ DF } & \multicolumn{2}{|c|}{ Plants $/ \mathrm{m}^{2}$} & \multicolumn{2}{|c|}{ Fodder yield } & \multicolumn{2}{|c|}{ Grain yield } & \multicolumn{2}{|c|}{ Pods/plant } & \multicolumn{2}{|c|}{ Grain/pod } & \multicolumn{2}{|c|}{ Wt/grain } \\
\hline & & & $F$ & $P$ & $F$ & $P$ & $F$ & $P$ & $F$ & $P$ & $F$ & $P$ & $F$ & $P$ \\
\hline \multirow[t]{7}{*}{1981} & Treatments & 23 & - & - & 1.31 & ns & 3.78 & $* * *$ & 1.85 & ns & 1.02 & ns & 0.97 & ns \\
\hline & Sole $v$ intercrop & 1 & - & - & 5.75 & $*$ & 50.35 & $* * *$ & 5.17 & * & 4.74 & $*$ & 0.79 & $\mathrm{~ns}$ \\
\hline & Sorghum genotypes & 22 & - & - & 1.11 & ns & 1.66 & ns & 1.70 & ns & 0.85 & ns & 0.98 & ns \\
\hline & Error & 46 & & & & & & & & & & & & \\
\hline & Intererop only & & & & & & & & & & & & & \\
\hline & Sorghum genotype & 22 & 0.91 & ns & 1.18 & ns & 2.43 & $* *$ & 2.06 & $*$ & 0.88 & ns & 1.03 & ns \\
\hline & Error & 44 & & & & & & & & & & & & \\
\hline \multirow[t]{6}{*}{1982} & Treatments & 14 & - & - & 2.43 & $*$ & 6.43 & $* * *$ & 2.37 & $*$ & 1.08 & ns & 0.68 & ns \\
\hline & Sole $v$ intercrop & 1 & - & - & 24.59 & $* * *$ & 49.73 & $* * *$ & 18.36 & $* * *$ & 0.01 & ns & 0.17 & ns \\
\hline & Sorghum genotype & 13 & - & - & 0.72 & ns & 3.09 & $* *$ & 1.14 & ns & 1.15 & ns & 0.72 & ns \\
\hline & Error & 26 & & & & & & & & & & & & \\
\hline & Intercrop only & 13 & 1.87 & ns & 1.14 & ns & 4.07 & $* *$ & 1.47 & ns & 1.08 & $\mathrm{~ns}$ & 0.68 & ns \\
\hline & Error & 26 & & & & & & & & & & & & \\
\hline
\end{tabular}

Intercropping caused a significant reduction in fodder and grain yield, pods/plant being the yield component mainly affected. However, plots of residuals against fitted values indicated that the error variances for sole and intercrop treatments might not be homogeneous, and an analysis confined to the intercrop cowpea treatments was also conducted. This raised the $F$ values for most of the variables, and those for grain yield and pods/ plant in 1981 became significant at the 5\% level. Thus sorghum genotypes differ in their effect on cowpea grain yield, but apparently not on fodder yield. This may have occurred because more cowpea leaves dropped before fodder harvest than is usual, due to a bacterial disease, Xanthomonas sp. (Singh and Allen, 1979). For grain yield and pods/plant, the variance is partitioned by both methods in Table 5, and the minimum, mean and maximum values and coefficients of variation of these characters are presented in Table 6. Dwarf and non-dwarf sorghum genotypes differ more in their effect on both cowpea characteristics than do sorghum genotypes bred by different methods.

\section{Regression analysis of intercrop variables on corresponding sole crop variables}

Scatter diagrams of each character measured in intercrop plotted against the same character measured in sole crop showed that there was a linear relationship between values in the two systems, and that the variation of the intercrop values was reasonably homogeneous at different sole crop values for all variables in both years. Lines of best fit were therefore calculated and their intercepts and slopes, and the significances of their deviations from zero, are presented in Table 7 . The slopes for all characters 
TABLE 5

Analysis of variance of cowpea plant characters in intercrop with the partition of sorghum genotype effects between groups bred by different methods, and between dwarf and non-dwarf types

\begin{tabular}{|c|c|c|c|c|c|c|}
\hline \multirow[t]{2}{*}{ Year } & \multirow{2}{*}{$\begin{array}{l}\text { Source of } \\
\text { variation }\end{array}$} & \multirow[t]{2}{*}{ DF } & \multicolumn{2}{|c|}{ Grain yield } & \multicolumn{2}{|c|}{ Pods/plant } \\
\hline & & & $F$ & $P$ & $F$ & $P$ \\
\hline \multirow[t]{10}{*}{1981} & Among groups & 3 & 3.93 & $*$ & 4.23 & $*$ \\
\hline & Sister lines & 11 & 0.97 & ns & 0.79 & ns \\
\hline & Improved lines & 5 & 5.05 & $* * *$ & 3.91 & $* *$ \\
\hline & Hybrids & 2 & 2.36 & ns & 0.71 & ns \\
\hline & Land races & 1 & 0.87 & ns & 0.18 & ns \\
\hline & Error & 44 & & & & \\
\hline & Among groups & 1 & 32.65 & $* * *$ & 23.21 & $* * *$ \\
\hline & Dwarf types & 2 & 1.02 & ns & 0.79 & ns \\
\hline & Non-dwarf types & 19 & 0.98 & ns & 1.08 & ns \\
\hline & Error & 44 & & & & \\
\hline \multirow[t]{10}{*}{1982} & Among groups & 3 & 2.41 & ns & 0.50 & ns \\
\hline & Sister lines & 3 & 0.95 & ns & 0.80 & ns \\
\hline & Improved lines & 4 & 6.70 & $* * *$ & 3.79 & $*$ \\
\hline & Hybrids & 2 & 3.58 & $*$ & 0.75 & ns \\
\hline & Land races & 1 & 8.85 & $* *$ & 0.12 & ns \\
\hline & Error & 26 & & & & \\
\hline & Among groups & 1 & 25.16 & $* * *$ & 7.30 & $*$ \\
\hline & Dwarf types & 2 & 3.52 & $*$ & 3.36 & * \\
\hline & Non-dwarf types & 10 & 2.07 & ns & 0.51 & ns \\
\hline & Error & 26 & & & & \\
\hline
\end{tabular}

TABLE 6

Minimum, mean and maximum sorghum-genotypes means, and coefficients of variation, of cow pea plant characters in intercrop

\begin{tabular}{|c|c|c|c|c|c|c|c|c|}
\hline \multirow[b]{3}{*}{ Character } & \multicolumn{8}{|c|}{ Year } \\
\hline & \multicolumn{4}{|l|}{1981} & \multicolumn{4}{|l|}{1982} \\
\hline & Min. & Mean & Max. & C.V. $(\%)$ & Min. & Mean & Max. & C.V. (\%) \\
\hline Grain yield $\left(\mathrm{g} / \mathrm{m}^{2}\right)$ & 17.1 & 22.6 & 33.0 & 19.1 & 13.2 & 15.1 & 19.4 & 14.4 \\
\hline Pods/plant & 2.53 & 3.37 & 4.43 & 17.3 & 1.77 & 2.31 & 3.17 & 25.5 \\
\hline
\end{tabular}

except heads/plant in 1981 were highly significantly different from zero, confirming other reports (e.g. Baker, 1974; Francis et al., 1978 a,b) that characters in intercropping are highly correlated with corresponding characters in sole cropping. However, correlation between characters in sole 
TABLE 7

Regressions of sorghum characters measured in intercrop on the corresponding characters measured in sole crop

\begin{tabular}{|c|c|c|c|c|c|c|}
\hline Year & Variable & Coefficient & Estimate & S.E. & $t$ & $P$ \\
\hline \multirow[t]{7}{*}{$\begin{array}{l}1981 \\
(D F=21)\end{array}$} & Canopy height & $\begin{array}{l}\text { Slope } \\
\text { Intercept }\end{array}$ & $\begin{array}{l}0.9585 \\
5.1862\end{array}$ & $\begin{array}{l}0.0270 \\
4.0\end{array}$ & $\begin{array}{r}35.11 \\
1.23\end{array}$ & $\begin{array}{l}* * * \\
\mathrm{~ns}\end{array}$ \\
\hline & Total leaf area & $\begin{array}{l}\text { Slope } \\
\text { Intercept }\end{array}$ & $\begin{array}{r}1.2549 \\
-164.3530\end{array}$ & $\begin{array}{l}0.1337 \\
313.0\end{array}$ & $\begin{array}{r}9.39 \\
-0.52\end{array}$ & $\begin{array}{l}* * * \\
\mathrm{~ns}\end{array}$ \\
\hline & Grain yield & $\begin{array}{l}\text { Slope } \\
\text { Intercept }\end{array}$ & $\begin{array}{c}0.554 \\
313.02\end{array}$ & $\begin{array}{l}0.0519 \\
150.0\end{array}$ & $\begin{array}{r}10.68 \\
2.09\end{array}$ & $\begin{array}{l}* * * \\
\mathrm{~ns}\end{array}$ \\
\hline & Dry fodder yield & $\begin{array}{l}\text { Slope } \\
\text { Intercept }\end{array}$ & $\begin{array}{c}0.6431 \\
-863.56\end{array}$ & $\begin{array}{l}0.0472 \\
327.0\end{array}$ & $\begin{array}{r}13.63 \\
-2.64\end{array}$ & $\begin{array}{l}* * * \\
*\end{array}$ \\
\hline & Heads/plant & $\begin{array}{l}\text { Slope } \\
\text { Intercept }\end{array}$ & $\begin{array}{l}0.2195 \\
0.8100\end{array}$ & $\begin{array}{l}0.1044 \\
0.0963\end{array}$ & $\begin{array}{l}2.10 \\
8.42\end{array}$ & $\begin{array}{l}\mathrm{ns} \\
* * *\end{array}$ \\
\hline & Grains/head & $\begin{array}{l}\text { Slope } \\
\text { Intercept }\end{array}$ & $\begin{array}{c}1.437 \\
147.84\end{array}$ & $\begin{array}{l}0.1492 \\
125.0\end{array}$ & $\begin{array}{l}9.63 \\
1.18\end{array}$ & $\begin{array}{l}* * * \\
\mathrm{~ns}\end{array}$ \\
\hline & Weight/grain & $\begin{array}{l}\text { Slope } \\
\text { Intercept }\end{array}$ & $\begin{array}{l}1.019 \\
1.417\end{array}$ & $\begin{array}{l}0.0486 \\
0.9\end{array}$ & $\begin{array}{r}20.94 \\
1.58\end{array}$ & $\begin{array}{l}* * * \\
\mathrm{~ns}\end{array}$ \\
\hline \multirow[t]{7}{*}{$\begin{array}{l}1982 \\
(\mathrm{DF}=12)\end{array}$} & Canopy height & $\begin{array}{l}\text { Slope } \\
\text { Intercept }\end{array}$ & $\begin{array}{l}0.9103 \\
6.0361\end{array}$ & $\begin{array}{l}0.050 \\
6.0\end{array}$ & $\begin{array}{r}18.21 \\
0.96\end{array}$ & $\begin{array}{l}* * * * \\
\mathrm{~ns}\end{array}$ \\
\hline & Total leaf area & $\begin{array}{l}\text { Slope } \\
\text { Intercept }\end{array}$ & $\begin{array}{r}1.1577 \\
55.0039\end{array}$ & $\begin{array}{l}0.1560 \\
469.0\end{array}$ & $\begin{array}{l}7.42 \\
0.12\end{array}$ & $\begin{array}{l}* * * \\
\mathrm{~ns}\end{array}$ \\
\hline & Grain yield & $\begin{array}{l}\text { Slope } \\
\text { Intercept }\end{array}$ & $\begin{array}{r}0.605 \\
-105.71\end{array}$ & $\begin{array}{l}0.0694 \\
360.0\end{array}$ & $\begin{array}{l}8.71 \\
0.29\end{array}$ & $\begin{array}{l}* * * \\
\mathrm{~ns}\end{array}$ \\
\hline & Dry fodder yield & $\begin{array}{l}\text { Slope } \\
\text { Intercept }\end{array}$ & $\begin{array}{r}0.6182 \\
-767.40\end{array}$ & $\begin{array}{l}0.0410 \\
353.0\end{array}$ & $\begin{array}{r}15.09 \\
-2.17\end{array}$ & $\begin{array}{l}* * * \\
*\end{array}$ \\
\hline & Heads/plant & $\begin{array}{l}\text { Slope } \\
\text { Intercept }\end{array}$ & $\begin{array}{r}2.049 \\
-1.001\end{array}$ & $\begin{array}{l}0.3644 \\
0.3760\end{array}$ & $\begin{array}{r}5.62 \\
-2.66\end{array}$ & $\begin{array}{l}* * * \\
*\end{array}$ \\
\hline & Grains/head & $\begin{array}{l}\text { Slope } \\
\text { Intercept }\end{array}$ & $\begin{array}{c}1.315 \\
201.63\end{array}$ & $\begin{array}{l}0.1231 \\
159.0\end{array}$ & $\begin{array}{r}10.68 \\
1.27\end{array}$ & $\begin{array}{l}* * * \\
\mathrm{~ns}\end{array}$ \\
\hline & Weight/grain & $\begin{array}{l}\text { Slope } \\
\text { Intercept }\end{array}$ & $\begin{array}{l}0.9108 \\
4.29\end{array}$ & $\begin{array}{l}0.1113 \\
2.5\end{array}$ & $\begin{array}{l}8.19 \\
1.74\end{array}$ & $\begin{array}{l}* * * \\
n s\end{array}$ \\
\hline
\end{tabular}

crop and in intercrop gives only their general relationship, whereas the breeder is interested in identifying genotypes which depart from the trend.

The intercepts were close to zero in all cases, differing at the $5 \%$ level of significance only for dry fodder yield and heads/plant in both years. This indicates that the intercrop/sole crop ratios, or their logarithms, are probably valid measures of a genotype's response to intercropping. Moreover the ratio for grain yield, i.e., the grain yield LER, is a widely used measure in intercrop studies (Willey, 1979; Mead and Riley, 1981). However since 
TABLE 8

Analysis of variance of the log (ratios) of sorghum plant characters with the partition of genotype effects among groups bred by different methods

\begin{tabular}{|c|c|c|c|c|c|c|c|}
\hline \multirow[b]{3}{*}{ Character } & \multirow[b]{3}{*}{$\begin{array}{l}\text { Source of } \\
\text { variation }\end{array}$} & \multicolumn{6}{|c|}{ Year } \\
\hline & & \multicolumn{3}{|c|}{1981} & \multicolumn{3}{|c|}{1982} \\
\hline & & DF & $F$ & $P$ & DF & $F$ & $P$ \\
\hline Plants $/ \mathrm{m}^{2}$ & $\begin{array}{l}\text { Genotypes } \\
\text { Among groups } \\
\text { Sister lines } \\
\text { Improved lines } \\
\text { Hybrids } \\
\text { Land races } \\
\text { Error }\end{array}$ & $\begin{array}{r}22 \\
3 \\
11 \\
5 \\
2 \\
1 \\
44\end{array}$ & $\begin{array}{l}1.87 \\
2.17 \\
1.97 \\
2.27 \\
0.75 \\
0.13\end{array}$ & $\begin{array}{l}\text { * } \\
\mathrm{ns} \\
\mathrm{ns} \\
\mathrm{ns} \\
\mathrm{ns} \\
\mathrm{ns}\end{array}$ & $\begin{array}{r}13 \\
3 \\
3 \\
4 \\
2 \\
1 \\
26\end{array}$ & $\begin{array}{l}2.22 \\
3.29 \\
1.91 \\
0.69 \\
4.71 \\
1.06\end{array}$ & $\begin{array}{l}* \\
* \\
\text { ns } \\
\text { ns } \\
* \\
\text { ns }\end{array}$ \\
\hline Canopy height & $\begin{array}{l}\text { Genotypes } \\
\text { Among groups } \\
\text { Sister lines } \\
\text { Improved lines } \\
\text { Hybrids } \\
\text { Land races } \\
\text { Error }\end{array}$ & $\begin{array}{r}22 \\
3 \\
11 \\
5 \\
2 \\
1 \\
44\end{array}$ & $\begin{array}{l}1.41 \\
0.58 \\
1.64 \\
2.06 \\
0.23 \\
0.46\end{array}$ & $\begin{array}{l}\text { ns } \\
\text { ns } \\
\text { ns } \\
\text { ns } \\
\text { ns } \\
\text { ns }\end{array}$ & $\begin{array}{r}13 \\
3 \\
3 \\
4 \\
2 \\
1 \\
26\end{array}$ & $\begin{array}{l}1.30 \\
3.15 \\
0.95 \\
0.26 \\
1.16 \\
0.69\end{array}$ & $\begin{array}{l}\text { ns } \\
* \\
\text { ns } \\
\text { ns } \\
\text { ns } \\
\text { ns }\end{array}$ \\
\hline Total leaf area & $\begin{array}{l}\text { Genotypes } \\
\text { Among groups } \\
\text { Sister lines } \\
\text { Improved lines } \\
\text { Hybrids } \\
\text { Land races } \\
\text { Error }\end{array}$ & $\begin{array}{r}22 \\
3 \\
11 \\
5 \\
2 \\
1 \\
44\end{array}$ & $\begin{array}{l}1.49 \\
1.78 \\
1.62 \\
1.44 \\
0.10 \\
2.25\end{array}$ & $\begin{array}{l}\text { ns } \\
\text { ns } \\
\text { ns } \\
\text { ns } \\
\text { ns } \\
\text { ns }\end{array}$ & $\begin{array}{r}13 \\
3 \\
3 \\
4 \\
2 \\
1 \\
26\end{array}$ & $\begin{array}{r}1.17 \\
0.78 \\
0.08 \\
0.33 \\
0.24 \\
10.34\end{array}$ & $\begin{array}{l}\text { ns } \\
\text { ns } \\
\text { ns } \\
\text { ns } \\
\text { ns } \\
* *\end{array}$ \\
\hline Dry fodder yield & $\begin{array}{l}\text { Genotypes } \\
\text { Among groups } \\
\text { Sister lines } \\
\text { Improved lines } \\
\text { Hybrids } \\
\text { Land races } \\
\text { Error }\end{array}$ & $\begin{array}{r}22 \\
3 \\
11 \\
5 \\
2 \\
1 \\
44\end{array}$ & $\begin{array}{l}2.89 \\
9.55 \\
1.33 \\
3.01 \\
2.52 \\
0.21\end{array}$ & $\begin{array}{l}* * \\
* * * \\
\text { ns } \\
* \\
\text { ns } \\
\text { ns }\end{array}$ & $\begin{array}{r}13 \\
3 \\
3 \\
4 \\
2 \\
1 \\
26\end{array}$ & $\begin{array}{l}3.19 \\
6.34 \\
2.47 \\
2.19 \\
1.15 \\
4.02\end{array}$ & $\begin{array}{l}* * * \\
* * \\
\mathrm{~ns} \\
\mathrm{~ns} \\
\mathrm{~ns} \\
\mathrm{~ns}\end{array}$ \\
\hline Grain yield & $\begin{array}{l}\text { Genotypes } \\
\text { Among groups } \\
\text { Sister lines } \\
\text { Improved lines } \\
\text { Hybrids } \\
\text { Land races } \\
\text { Error }\end{array}$ & $\begin{array}{r}22 \\
3 \\
11 \\
5 \\
2 \\
1 \\
44\end{array}$ & $\begin{array}{l}2.77 \\
7.49 \\
1.92 \\
2.72 \\
0.57 \\
2.55\end{array}$ & $\begin{array}{l}* * \\
* * * \\
\mathrm{~ns} \\
* \\
\mathrm{~ns} \\
\mathrm{~ns}\end{array}$ & $\begin{array}{r}13 \\
3 \\
3 \\
4 \\
2 \\
1 \\
26\end{array}$ & $\begin{array}{l}1.26 \\
1.82 \\
1.24 \\
1.36 \\
0.20 \\
1.31\end{array}$ & $\begin{array}{l}\text { ns } \\
\text { ns } \\
\text { ns } \\
\text { ns } \\
\text { ns } \\
\text { ns }\end{array}$ \\
\hline Heads/plant & $\begin{array}{l}\text { Genotypes } \\
\text { Among groups } \\
\text { Sister lines } \\
\text { Improved lines } \\
\text { Hybrids } \\
\text { Land races } \\
\text { Error }\end{array}$ & $\begin{array}{r}22 \\
3 \\
11 \\
5 \\
2 \\
1 \\
44\end{array}$ & $\begin{array}{r}1.43 \\
0.57 \\
0.78 \\
0.64 \\
0.56 \\
16.87\end{array}$ & $\begin{array}{l}\text { ns } \\
\text { ns } \\
\text { ns } \\
\text { ns } \\
* * * \\
* * *\end{array}$ & $\begin{array}{r}13 \\
3 \\
3 \\
4 \\
1 \\
1 \\
26\end{array}$ & $\begin{array}{l}2.54 \\
7.31 \\
0.04 \\
0.65 \\
0.42 \\
7.51\end{array}$ & $\begin{array}{l}* \\
* * \\
\mathrm{~ns} \\
\mathrm{~ns} \\
\mathrm{~ns} \\
*\end{array}$ \\
\hline
\end{tabular}


TABLE 8 (cont.)

\begin{tabular}{|c|c|c|c|c|c|c|c|}
\hline \multirow[b]{3}{*}{ Character } & \multirow[b]{3}{*}{$\begin{array}{l}\text { Source of } \\
\text { variation }\end{array}$} & \multicolumn{6}{|c|}{ Year } \\
\hline & & \multicolumn{3}{|c|}{1981} & \multicolumn{3}{|c|}{1982} \\
\hline & & $\mathrm{DF}$ & $F$ & $P$ & $\mathrm{DF}$ & $F$ & $P$ \\
\hline \multirow[t]{7}{*}{ Grain Head } & Genotypes & 22 & 1.05 & ns & 13 & 3.12 & $* *$ \\
\hline & Among groups & 3 & 1.47 & ns & 3 & 5.34 & $*$ \\
\hline & Sister lines & 11 & 1.47 & ns & 3 & 1.61 & $\mathrm{~ns}$ \\
\hline & Improved lines & 5 & 0.49 & ns & 4 & 4.41 & $* *$ \\
\hline & Hybrids & 2 & 0.00 & ns & 2 & 0.58 & ns \\
\hline & Land races & 1 & 0.08 & $\mathrm{~ns}$ & 1 & 0.83 & ns \\
\hline & Error & 44 & & & 21 & & \\
\hline \multirow[t]{7}{*}{ Weight grain } & Genotypes & 22 & 0.69 & ns & 13 & 6.92 & $* * *$ \\
\hline & Among groups & 3 & 0.35 & ns & 3 & 12.41 & $* * *$ \\
\hline & Sister lines & 11 & 0.58 & ns & 3 & 3.64 & $*$ \\
\hline & Improved lines & 5 & 1.19 & ns & 4 & 5.96 & $* *$ \\
\hline & Hybrids & 2 & 0.77 & ns & 2 & 0.86 & ns \\
\hline & Land races & 1 & 0.12 & ns & 1 & 16.23 & $* * *$ \\
\hline & Error & 44 & & & 26 & & \\
\hline
\end{tabular}

the slopes are mostly very different from 1 , the genotype $X$ cropping system interaction effect would not be a valid alternative.

\section{Analysis of the intercrop/sole crop ratios}

Plots of residuals against fitted values (Draper and Smith, 1981) indicated that the $\log$ (ratios) had less heterogeneity of variance than the ratios for most characters, and were sufficiently homogenous to proceed with analyses of variance, which are presented in Table 8 , with the variance partitioned between sister lines, improved lines, hybrids and land races, and in Table 9 with the vafiance partitioned between dwarf and non-dwarf groups of genotypes. In Table 10 the minimum, mean and maximum ratios (obtained by back transforming the log (ratios) and the standard error factors (obtained by back transforming the SEs of the mean log (ratios)) are presented. The coefficient of variation is not an appropriate measure of experimental precision in this case, since negative log (ratios) can occur. The $F$ values are generally lower than those for analysis within cropping systems, confirming that much of the genetic variation was common to the two systems. However there was significant genetic variation for some characters in both years, including grain and fodder yields, though the variation for fodder yield and heads/plant may have been due to the non-zero intercept of the intercrop-sole crop regression for these characters. Hence selection for performance of the sorghum component of the intercrop, leaving aside its effect on the associated cowpea, can largely be conducted in sole crop, 


\section{TABLE 9}

Analyses of variance of the log (ratios) of sorghum plant characters with the partition of genotypes effects among dwarf and non-dwarf types

\begin{tabular}{|c|c|c|c|c|c|c|c|}
\hline \multirow[b]{3}{*}{ Character } & \multirow[b]{3}{*}{$\begin{array}{l}\text { Source of } \\
\text { variation }\end{array}$} & \multicolumn{6}{|c|}{ Year } \\
\hline & & \multicolumn{3}{|c|}{1981} & \multicolumn{3}{|c|}{1982} \\
\hline & & DF & $F$ & $P$ & DF & $F$ & $P$ \\
\hline Plants $/ \mathrm{m}^{2}$ & $\begin{array}{l}\text { Among groups } \\
\text { Dwarf types } \\
\text { Non-dwarf types } \\
\text { Error }\end{array}$ & $\begin{array}{r}1 \\
2 \\
19 \\
4\end{array}$ & $\begin{array}{l}9.67 \\
0.24 \\
1.63\end{array}$ & $\begin{array}{l}* * \\
\mathrm{~ns} \\
\mathrm{~ns}\end{array}$ & $\begin{array}{r}1 \\
2 \\
10 \\
26\end{array}$ & $\begin{array}{l}7.18 \\
1.08 \\
1.95\end{array}$ & $\begin{array}{l}* \\
\text { ns } \\
\text { ns }\end{array}$ \\
\hline Canopy height & $\begin{array}{l}\text { Among groups } \\
\text { Dwarf types } \\
\text { Non-dwarf types } \\
\text { Error }\end{array}$ & $\begin{array}{r}1 \\
2 \\
19 \\
44\end{array}$ & $\begin{array}{l}0.12 \\
1.32 \\
1.49\end{array}$ & $\begin{array}{l}\mathrm{ns} \\
\mathrm{ns} \\
\mathrm{ns}\end{array}$ & $\begin{array}{r}1 \\
2 \\
10 \\
26\end{array}$ & $\begin{array}{l}0.01 \\
0.28 \\
1.58\end{array}$ & $\begin{array}{l}\text { ns } \\
\text { ns } \\
\text { ns }\end{array}$ \\
\hline Total leaf area & $\begin{array}{l}\text { Among groups } \\
\text { Dwarf types } \\
\text { Non-dwarf types } \\
\text { Error }\end{array}$ & $\begin{array}{r}1 \\
2 \\
19 \\
44\end{array}$ & $\begin{array}{r}12.53 \\
1.26 \\
0.93\end{array}$ & $\begin{array}{l}* * * \\
\mathrm{~ns} \\
\mathrm{~ns}\end{array}$ & $\begin{array}{r}1 \\
2 \\
10 \\
26\end{array}$ & $\begin{array}{l}0.46 \\
0.28 \\
1.37\end{array}$ & $\begin{array}{l}\text { ns } \\
\text { ns } \\
\text { ns }\end{array}$ \\
\hline Dry fodder yield & $\begin{array}{l}\text { Among groups } \\
\text { Dwarf types } \\
\text { Non-dwarf types } \\
\text { Error }\end{array}$ & $\begin{array}{r}1 \\
2 \\
19 \\
44\end{array}$ & $\begin{array}{r}22.46 \\
1.96 \\
1.96\end{array}$ & $\begin{array}{l}* * \\
\text { ns } \\
\text { ns }\end{array}$ & $\begin{array}{r}1 \\
2 \\
10 \\
26\end{array}$ & $\begin{array}{r}16.43 \\
0.46 \\
2.42\end{array}$ & $\begin{array}{l}* * * \\
\text { ns } \\
*\end{array}$ \\
\hline Grain yield & $\begin{array}{l}\text { Among groups } \\
\text { Dwarf types } \\
\text { Non-dwarf types } \\
\text { Error }\end{array}$ & $\begin{array}{r}1 \\
2 \\
19 \\
44\end{array}$ & $\begin{array}{r}32.78 \\
0.51 \\
1.43\end{array}$ & $\begin{array}{l}* * \\
\mathrm{~ns} \\
\mathrm{~ns}\end{array}$ & $\begin{array}{r}1 \\
2 \\
10 \\
26\end{array}$ & $\begin{array}{l}9.47 \\
0.59 \\
0.57\end{array}$ & $\begin{array}{l}* * \\
\mathrm{~ns} \\
\mathrm{~ns}\end{array}$ \\
\hline Heads/plant & $\begin{array}{l}\text { Among groups } \\
\text { Dwarf types } \\
\text { Non-dwarf types } \\
\text { Error }\end{array}$ & $\begin{array}{r}1 \\
2 \\
19 \\
44\end{array}$ & $\begin{array}{l}0.00 \\
2.01 \\
1.45\end{array}$ & $\begin{array}{l}\mathrm{ns} \\
\mathrm{ns} \\
\mathrm{ns}\end{array}$ & $\begin{array}{r}1 \\
2 \\
10 \\
26\end{array}$ & $\begin{array}{l}0.01 \\
1.30 \\
3.04\end{array}$ & $\begin{array}{l}\mathrm{ns} \\
\mathrm{ns} \\
*\end{array}$ \\
\hline Grains/head & $\begin{array}{l}\text { Among groups } \\
\text { Dwarf types } \\
\text { Non-dwarf types } \\
\text { Error }\end{array}$ & $\begin{array}{r}1 \\
2 \\
19 \\
44\end{array}$ & $\begin{array}{l}8.29 \\
0.03 \\
0.78\end{array}$ & $\begin{array}{l}* * \\
\mathrm{~ns} \\
\mathrm{~ns}\end{array}$ & $\begin{array}{r}1 \\
2 \\
10 \\
26\end{array}$ & $\begin{array}{r}24.37 \\
2.11 \\
1.19\end{array}$ & $\begin{array}{l}* * * \\
\mathrm{~ns} \\
\mathrm{~ns}\end{array}$ \\
\hline Weight/grain & $\begin{array}{l}\text { Among groups } \\
\text { Dwarf types } \\
\text { Non-dwarf types } \\
\text { Error }\end{array}$ & $\begin{array}{r}1 \\
2 \\
19 \\
44\end{array}$ & $\begin{array}{l}0.11 \\
0.68 \\
1.06\end{array}$ & $\begin{array}{l}\mathrm{ns} \\
\mathrm{ns} \\
\mathrm{ns}\end{array}$ & $\begin{array}{r}1 \\
2 \\
10 \\
26\end{array}$ & $\begin{array}{r}26.75 \\
5.48 \\
5.23\end{array}$ & $\begin{array}{l}* * * \\
* * \\
* * *\end{array}$ \\
\hline
\end{tabular}

but even here there is some scope for specific selection for intercropping. The partitioning into dwarf and non-dwarf groups produced higher betweengroups $F$ ratios in almost every case with the exception of canopy height: this character appears to be so stable that there is almost no genotypic 
TABLE 10

Minimum, mean and maximum genotype means and standard error factors for sole crop/intercrop ratios of sorghum plant characters

\begin{tabular}{|c|c|c|c|c|c|c|c|c|}
\hline \multirow[b]{3}{*}{ Character } & \multicolumn{8}{|l|}{ Year } \\
\hline & \multicolumn{4}{|l|}{1981} & \multicolumn{4}{|l|}{1982} \\
\hline & Min. & Mean & Max. & $\begin{array}{l}\times \text { S.E. } \\
\text { factor }\end{array}$ & Min. & Mean & Max. & $\begin{array}{l}\times \text { S.E. } \\
\text { factor }\end{array}$ \\
\hline Plants $/ m^{2}$ & 0.309 & 0.325 & 0.351 & 1.051 & 0.319 & 0.328 & 0.343 & 1.032 \\
\hline Canopy height & 0.418 & 0.434 & 0.450 & 1.037 & 0.412 & 0.427 & 0.444 & 1.041 \\
\hline Total leaf area & 0.413 & 0.466 & 0.517 & 1.104 & 0.391 & 0.460 & 0.514 & 1.148 \\
\hline Dry fodder yield & 0.279 & 0.322 & 0.354 & 1.081 & 0.302 & 0.326 & 0.352 & 1.061 \\
\hline Grain yield & 0.286 & 0.375 & 0.456 & 1.169 & 0.318 & 0.343 & 0.363 & 1.082 \\
\hline Heads/plant & 0.410 & 0.456 & 0.539 & 1.115 & 0.431 & 0.447 & 0.510 & 1.064 \\
\hline Grains/head & 0.286 & 0.375 & 0.456 & 1.235 & 0.455 & 0.521 & 0.573 & 1.079 \\
\hline Weight/grain & 0.440 & 0.452 & 0.468 & 1.052 & 0.419 & 0.455 & 0.485 & 1.035 \\
\hline
\end{tabular}

variation in its log (ratio). Thus the method by which a genotype was bred, which is important in explaining the variation between genotypes in either system, becomes less important than the non-dwarf/dwarf grouping when the response of genotypes to the cropping system is considered. The sole crop values of the characters measured range from about a third to a half of their intercrop values, and it is notable that even weight/grain is highly responsive to the cropping system.

\section{Sorghum yield related to sorghum plant characters}

The univariate regressions of the sorghum intercrop yield on sorghum variables measured in sole crop are presented in Table 11. They indicate that the intercrop yield was primarily a function of grains/head. Days to flowering and weight/grain had some influence in 1981 but not in 1982 .

The multiple regression models produced by the stepwise method with all the variables recorded in sole crop available for inclusion, and with a critical value of $t=1.5$, are as follows:

\section{Sorghum}

1981: intercrop $=-42.5+0.0605 \times$ grains $/$ head yield $\left(\mathrm{g} / \mathrm{m}^{2}\right)+1.67 \times$ weight/grain $(\mathrm{mg})$

$\mathrm{F}_{3,19}=47.78 * *$

$+0.0902 \times$ canopy height $(\mathrm{cm})$

\section{Sorghum}

1982: intercrop $=-73.4+0.0691 \times$ grains $/$ head yield $\left(\mathrm{g} / \mathrm{m}^{2}\right) \quad+3.78 \times$ weight/grain $(\mathrm{mg})$

$\mathrm{F}_{2,11}=30.14 * *$ 
TABLE 11

Univariate regressions of sorghum intercrop yield $\left(\mathrm{g} / \mathrm{m}^{2}\right)$ on sorghum characters measured in sole crop

\begin{tabular}{|c|c|c|c|c|c|c|}
\hline \multirow{2}{*}{$\begin{array}{l}\text { Year } \\
\text { Variable }\end{array}$} & \multicolumn{3}{|c|}{$1981(\mathrm{DF}=21)$} & \multicolumn{3}{|c|}{$1982(\mathrm{DF}=12)$} \\
\hline & $b$ & $t$ & $P$ & $b$ & $t$ & $P$ \\
\hline Canopy height $(\mathrm{cm})$ & 0.020 & 0.18 & $\mathrm{~ns}$ & -0.091 & -0.63 & ns \\
\hline Total leaf area $\left(\mathrm{cm}^{2}\right)$ & -0.0067 & -0.62 & ns & -0.00370 & -0.44 & ns \\
\hline Days to flowering & -1.75 & -2.42 & $*$ & -0.570 & -0.47 & ns \\
\hline Heads/plant & 62.3 & 1.67 & ns & -58.66 & -0.73 & ns \\
\hline Grains/head & 0.0609 & 7.91 & $* * *$ & 0.0386 & 4.83 & $* * *$ \\
\hline Weight/grain (mg) & 3.18 & 2.61 & $*$ & 2.13 & -1.67 & ns \\
\hline
\end{tabular}

The variables grains/head and weight/grain were important predictors in both years, but canopy height was important only in 1981. When a new variable is included in a regression model it is to be expected that the coefficients of the other variables will change, and taking this into account the coefficients were fairly similar in the two seasons.

An analysis of variance of genotype effects after allowing for effects of the variables retained in the models for each year is presented in Table 12. Genetic variation significant at the $5 \%$ level could still be detected in both years, indicating that a genotype's potential in intercrop could not be entirely predicted from these characters measured in sole crop.

TABLE 12

Analysis of variance of genotype effects on intercrop yield after allowing for effects of variables measured in sole crop and retained in the model in 1981 and 1982 , respectively

\begin{tabular}{|c|c|c|c|c|c|}
\hline Year & Source of variation & DF & $\mathrm{MS}^{\mathrm{a}}$ & $F$ & $P$ \\
\hline \multirow[t]{6}{*}{1981} & Replication & 2 & 89.97 & 0.85 & ns \\
\hline & Grains/head & 1 & 27793 & 261.08 & $* * *$ \\
\hline & Weight/grain & 1 & 4552 & 42.76 & $* * *$ \\
\hline & Canopy/height & 1 & 742.6 & 6.98 & $* *$ \\
\hline & Genotypes & 22 & 194.9 & 1.83 & $*$ \\
\hline & Residual & 41 & 106.5 & & \\
\hline \multirow[t]{5}{*}{1982} & Replication & 2 & 1303 & 10.68 & $* * *$ \\
\hline & Grains/head & 1 & 14527 & 119.11 & $* * *$ \\
\hline & Weight/grain & 1 & 3593 & 29.46 & $* * *$ \\
\hline & Genotypes & 13 & 321.5 & 2.64 & $*$ \\
\hline & Residual & 24 & 121.8 & & \\
\hline
\end{tabular}

a This column is obtained from the reduction in residual SS due to the successive inclusion of each term, divided by the DF for the term. 
The next step is to determine whether a better prediction can be made from measurements in intercrop.

The univariate regression analyses of sorghum intercrop yield on sorghum variables in intercropping, with $t$-values and their significance levels, are presented in Table 13. As before, grains/head was the most influential character, with weight/grain having some effect in 1981 . The models obtained from stepwise regression with all sorghum characters measured in intercrop available for inclusion are as follows:

\section{Sorghum}

1981: intercrop $=-65.6+0.0376 \times$ grains $/$ head yield $\left(\mathrm{g} / \mathrm{m}^{2}\right)+1.84 \times$ weight/grain $(\mathrm{mg})$

$\mathrm{F}_{3,19}=149.12 * * *$

$$
+29.4 \times \text { heads/plant }
$$

\section{Sorghum}

1982: intercrop $=-122+0.0468 \times \mathrm{grain} / \mathrm{head}$

yield $\left(\mathrm{g} / \mathrm{m}^{2}\right)+4.21 \times$ weight/grain $(\mathrm{mg})$

$+0.00284 \times$ total leaf area $\left(\mathrm{cm}^{2}\right)$

$+2.82 \times$ time of achievement of max. canopy

width (days)

$\mathrm{F}_{5,8}=185.90 * * *$

$-0.0369 \times$ maximum canopy height $(\mathrm{cm})$

\section{TABLE 13}

Univariate regressions of sorghum intercrop yield $\left(\mathrm{g} / \mathrm{m}^{2}\right)$ on sorghum characters measured in intercrop

\begin{tabular}{|c|c|c|c|c|c|c|}
\hline \multirow{2}{*}{$\begin{array}{l}\text { Year } \\
\text { Variable }\end{array}$} & \multicolumn{3}{|c|}{$1981(\mathrm{DF}=21)$} & \multicolumn{3}{|c|}{$1982(\mathrm{DF}=12)$} \\
\hline & $b$ & $t$ & $P$ & $b$ & $t$ & $P$ \\
\hline Maximum canopy height $(\mathrm{cm})$ & 0.0126 & 0.12 & ns & -0.00275 & 0.62 & ns \\
\hline $\begin{array}{l}\text { Time of achievement of } \\
\text { maximum canopy height }(\mathrm{cm})\end{array}$ & 0.552 & 0.27 & ns & 0.116 & 0.58 & ns \\
\hline $\begin{array}{l}\text { Rate of achievement of } \\
\text { maximum canopy height }(\mathrm{cm} / \mathrm{day})\end{array}$ & -0.408 & -0.60 & ns & -0.0188 & -0.63 & $n s$ \\
\hline Maximum canopy width $(\mathrm{cm})$ & -0.0854 & -0.22 & ns & -0.00701 & -0.40 & ns \\
\hline $\begin{array}{l}\text { Time of achievement of } \\
\text { maximum canopy width (days) }\end{array}$ & 1.85 & 0.79 & ns & 0.140 & 1.08 & ns \\
\hline $\begin{array}{l}\text { Rate of achievement of } \\
\text { maximum canopy width }(\mathrm{cm} / \text { day })\end{array}$ & -0.508 & -0.55 & ns & -1.200 & -1.51 & ns \\
\hline Total leaf area $\left(\mathrm{cm}^{2}\right)$ & -0.00852 & -1.12 & ns & 0.00167 & -0.23 & ns \\
\hline Heads/plant & 77.2 & 1.05 & ns & -1.30 & -1.31 & ns \\
\hline Grains/head & 0.0413 & 11.84 & $* * *$ & 0.000938 & 6.57 & $* * *$ \\
\hline Weight/grain (mg) & 3.07 & 2.63 & $*$ & -0.0474 & -1.13 & ns \\
\hline
\end{tabular}


Only grains/head and weight/grain entered the model in both years, but they entered with fairly similar coefficients in the two years. Canopy characteristics were important in 1982 but not in 1981 .

The analysis of variance of genotype effects after allowing for the effect of variables retained in the models (Table 14) showed that genetic variation, significant at the $5 \%$ level in 1981 and at the $1 \%$ level in 1982 , was still unaccounted for. This indicates the existence of some variable under genetic control but not included in the model.

Comparison of the multivariate regression models shows that grains/head and weight/grain, measured either in sole or in intercrop, were consistent predictors of the sorghum intercrop yield. However comparison of Tables 12 and 14 shows that both genotype and residual mean squares were lower when the model was based on variables measured in intercrop, indicating that a more precise prediction of yield can be obtained by evaluating a genotype in this system. This may be partly an artefact because the intercrop yield was measured in the same plots as the other intercrop characters.

TABLE 14

Analysis of variance of genotype effects on intercrop yield after allowing for effects of variables measured in intercrop and retained in the model in 1981 and 1982, re. spectively

\begin{tabular}{|c|c|c|c|c|c|}
\hline Year & Source of variation & DF & MS & $F$ & $P$ \\
\hline \multirow[t]{6}{*}{1981} & Replication & 2 & 89.97 & 2.42 & ns \\
\hline & Grains/head & 1 & 32130 & 865.39 & $* * *$ \\
\hline & Weight/grain & 1 & 4517 & 121.66 & $* * *$ \\
\hline & Heads/plant & 1 & 1775 & 47.81 & $* * *$ \\
\hline & Genotypes & 22 & 81.94 & 2.21 & * \\
\hline & Residual & 41 & 37.12 & & \\
\hline \multirow[t]{8}{*}{1982} & Replication & 2 & 1303 & 73.39 & $* * *$ \\
\hline & Grains/head & 1 & 18652 & 1097.00 & $* * *$ \\
\hline & Weight/grain & 1 & 5137 & 302.33 & $* * *$ \\
\hline & Total leaf area & 1 & 2.289 & 0.13 & ns \\
\hline & $\begin{array}{l}\text { Maximum canopy height } \\
\text { Time of achieving }\end{array}$ & 1 & 7.200 & 0.42 & ns \\
\hline & maximum canopy width & 1 & 43.61 & 2.57 & ns \\
\hline & Genotypes & 13 & 76.95 & 5.31 & $* * *$ \\
\hline & Residual & 23 & 17.76 & & \\
\hline
\end{tabular}

\section{Cowpea intercrop grain yield related to sorghum plant characters}

The univariate regression of cowpea intercrop grain yield on sole crop sorghum characters are presented in Table 15. Cowpea intercrop yield was negatively associated with the related characters canopy height, total leaf area and fodder yield in 1981 but these associations were weaker or absent in 1982 . 
TABLE 15

Univariate regressions of cowpea intercrop yield $\left(\mathrm{g} / \mathrm{m}^{2}\right)$ on sorghum characters measured in sole crop

\begin{tabular}{|c|c|c|c|c|c|c|}
\hline \multirow{2}{*}{$\begin{array}{l}\text { Year } \\
\text { Variable }\end{array}$} & \multicolumn{3}{|c|}{$1981(\mathrm{DF}=21)$} & \multicolumn{3}{|c|}{$1982(\mathrm{DF}=12)$} \\
\hline & $b$ & $t$ & $P$ & $b$ & $t$ & $P$ \\
\hline Canopy height (cm) & -0.0386 & -2.41 & * & 0.0111 & 0.50 & ns \\
\hline Total leaf area $\left(\mathrm{cm}^{2}\right)$ & -0.00520 & -3.51 & $* *$ & 0.000561 & 0.62 & ns \\
\hline Grain yield $\left(\mathrm{g} / \mathrm{m}^{2}\right)$ & 0.0232 & 1.06 & ns & 0.0023 & 0.11 & ns \\
\hline Dry fodder yield $\left(\mathrm{g} / \mathrm{m}^{2}\right)$ & -0.0463 & -3.07 & $* *$ & -0.0192 & -2.59 & $*$ \\
\hline Heads/plant & 9.75 & 1.52 & ns & 18.5 & 2.53 & $*$ \\
\hline Grains/head & 0.00161 & 0.62 & $\mathrm{~ns}$ & -0.000210 & -0.14 & ns \\
\hline Weight/grain (mg) & 0.151 & 0.63 & ns & -0.0230 & -0.15 & ns \\
\hline
\end{tabular}

The models obtained from stepwise regression of cowpea intercrop yield on sorghum characters measured in sole crop area as follows:

\section{Cowpea}

1981: intercrop $=37.5-0.00459 \times$ total leaf area $\left(\mathrm{cm}^{2}\right)$ yield $\left(\mathrm{g} / \mathrm{m}^{2}\right) \quad-0.0279 \times$ canopy height $(\mathrm{cm})$

$\mathrm{F}_{2,20}=9.64 * *$

\section{Cowpea}

1982: intercrop $=-4.63+23.8 \times$ heads $/$ plant

$\mathrm{F}_{2,10}=4.78^{*}$ yield $\left(\mathrm{g} / \mathrm{m}^{2}\right) \quad-0.205$ weight/grain $(\mathrm{mg})$

The cowpea intercrop yield was predicted by variables related to light transmission to the cowpea in 1981 but only a weak prediction, based on yield components, was found in 1982 .

The univariate regressions of cowpea intercrop yield on sorghum characters measured in intercrop are presented in Table 16. In 1981 there was a positive and highly significant slope for the regression on percentage of light transmission and consequently the sorghum characters that prevent light reaching the cowpea, e.g. canopy width, total leaf area and canopy height, had negative and significant slopes as did fodder yield. Similar but weaker trends were found in 1982 . High association between the intercrop legume yield and the avallability of light has also been demonstrated by Gardner and Cracker (1981) and Marshall and Willey (1983).

The models obtained from stepwise regression of cowpea intercrop yield on sorghum characters measured in intercrop are as follows:

\section{Cowpea}

1981: intercrop $\quad=\quad 6.94+0.248 \times$ light transmission $(\%)$

yield $\left(\mathrm{g} / \mathrm{m}^{2}\right) \quad-0.00172 \times$ total leaf area $\left(\mathrm{cm}^{2}\right)$

$+14.1 \times$ heads/plant +0.00395

$\mathrm{F}_{4,18}=14.67 * * * \quad \times$ canopy height $(\mathrm{cm})$ 
TABLE 16

Univariate regressions of cowpea intercrop yield on sorghum characters measured in intercrop

\begin{tabular}{|c|c|c|c|c|c|c|}
\hline \multirow{2}{*}{$\begin{array}{l}\text { Year } \\
\text { Variable }\end{array}$} & \multicolumn{3}{|c|}{$1981(\mathrm{DF}=21)$} & \multicolumn{3}{|c|}{$1982(\mathrm{DF}=12)$} \\
\hline & $b$ & $t$ & $P$ & $b$ & $t$ & $P$ \\
\hline Canopy height $(\mathrm{cm})$ & -0.0422 & -2.63 & $*$ & 0.0254 & -1.78 & ns \\
\hline Canopy width $(\mathrm{cm})$ & -0.179 & -3.25 & $* *$ & -0.188 & -5.72 & $* * *$ \\
\hline Light transmission (\%) & 0.183 & 4.89 & $* * *$ & 0.118 & 3.92 & $* * *$ \\
\hline Total leaf area $\left(\mathrm{cm}^{2}\right)$ & -0.00425 & -4.40 & $* * *$ & 0.00092 & 1.46 & ns \\
\hline Grain yield $\left(\mathrm{g} / \mathrm{m}^{2}\right)$ & -0.02 & -0.55 & ns & -0.02 & -0.57 & ns \\
\hline Dry fodder yield $\left(\mathrm{g} / \mathrm{m}^{2}\right)$ & -0.077 & -3.73 & $* *$ & -0.03 & -2.56 & $*$ \\
\hline Heads/plant & 19.6 & 0.23 & ns & 6.65 & 2.06 & ns \\
\hline Grains/head & -0.00083 & -0.51 & $n s$ & -0.00957 & -0.91 & ns \\
\hline Weight/grain (mg) & 0.0703 & 0.31 & ns & 0.133 & 0.87 & ns \\
\hline
\end{tabular}

\section{Cowpea}

1982: intercrop $=46.4-0.130 \times$ canopy width $(\mathrm{cm})$

yield $\left(\mathrm{g} / \mathrm{m}^{2}\right) \quad-0.00181 \times$ grains $/$ head

$-0.222 \times$ days to flowering

$-0.212 \times$ weight/grain $(g)$

$\mathrm{F}_{3,9}=17.39 * * *$

The presence of entirely different, though related, variables in the two models indicates that sorghum characters were not consistent in their effect on cowpea intercrop yields in different years.

More precise predictions of the intercrop cowpea grain yield can be obtained from measurements of the sorghum canopy structure in intercrop than from measurements in sole crop, and more variables are involved in the predictions. This indicates that sorghum variables measured at sole crop density are not sufficient to explain cowpea intercrop yield, contrary to the findings of Baker and Yusuf (1976).

The analyses of variance of sorghum genotype effects on cowpea intercrop yield after allowing for the effects of variables retained in the 1981 and 1982 models are presented in Table 17. No genetic variation was left unaccounted for in 1981 but an effect of sorghum genotype significant at the $5 \%$ level was found in 1982 . The significant $F$-value is a reflection of a better fit of the whole model in 1982, since both genotype and residual mean squares were lower in this season. It indicates that some character of the sorghum under genetic control but not included in the model influences cowpea yield.

Since cowpea intercrop yield was highly positively associated with percentage of transmitted light in both years, the sorghum characters that affect the amount of light reaching the cowpea are relevant to the improvement of intercrop cowpea yield. These characters cannot be mea- 
sured at sole crop density, but could perhaps be measured at intercrop density even in the absence of cowpea if it can be argued that cowpea has little competitive effect on the sorghum. If so, good sorghum canopies for intercropping may be selected as early as the $F_{2}$ generation, commonly grown at low density.

TABLE 17

Analysis of variance of genotype effects on intercrop cowpea yield after allowing for effects of sorghum characters measured in intercrop and retained in the model in 1981 and 1982 respectively

\begin{tabular}{|c|c|c|c|c|c|}
\hline Year & Source of variation & DF & MS & $F$ & $P$ \\
\hline \multirow[t]{7}{*}{1981} & Replication & 2 & 47.18 & 4.20 & $*$ \\
\hline & Total leaf area & 1 & 216.0 & 19.22 & $* * *$ \\
\hline & Light transmission & 1 & 689.1 & 61.34 & $* * *$ \\
\hline & Heads/plant & 1 & 67.56 & 6.01 & $*$ \\
\hline & Canopy height & 1 & 75.29 & 6.70 & $*$ \\
\hline & Sorghum genotype & 22 & 14.54 & 1.29 & ns \\
\hline & Residual & 40 & 11.23 & & \\
\hline \multirow[t]{7}{*}{1982} & Replication & 2 & 7.997 & 2.14 & ns \\
\hline & Canopy width & 1 & 152.3 & 40.76 & $* * *$ \\
\hline & Grains/head & 1 & 10.53 & 2.82 & $\mathrm{~ns}$ \\
\hline & Days to flowering & 1 & 38.34 & 10.26 & $* *$ \\
\hline & Weight/grain & 1 & 3.592 & 0.96 & $\mathrm{~ns}$ \\
\hline & Sorghum genotypes & 12 & 8.391 & 2.25 & $*$ \\
\hline & Residual & 23 & 3.737 & & \\
\hline
\end{tabular}

Land equivalent ratios related to sorghum plant characters

The genotype mean values of sorghum and cowpea yields and LERs (untransformed), the total LERs given by

$$
\frac{\text { sorghum intercrop yield }}{\text { sorghum sole crop yield }}+\frac{\text { cowpea intercrop yield }}{\text { cowpea sole crop yield }}
$$

and some of the characters shown to be important in the foregoing analyses are presented in Table 18. The sorghum LERs were always above their expected value of 0.33 but in both years the dwarf genotypes (S1021, 2219B, M66433) consistently gave lower LERs than the non-dwarf types. The cowpea LERs were often below their expected value of 0.67 , and were about the same in both years, despite different cowpea yield levels. Total LERs above 1.0 were obtained for all genotypes in both years. This indicates that a higher yield can be obtained from growing the intercrop than from the sole sorghum and sole cowpea grown separately. Trenbath (1976) suggested that a total LER greater than 1.00 indicates that the 
Mean yield and LERs, and influential sorghum plant characters

\begin{tabular}{|c|c|c|c|c|c|c|c|c|c|c|c|c|}
\hline \multirow[t]{3}{*}{ Yeax } & \multirow[t]{3}{*}{ Genotype } & \multirow{3}{*}{$\begin{array}{l}\text { Height } \\
\text { (cm) }\end{array}$} & \multirow{3}{*}{$\begin{array}{l}\text { Width } \\
\text { (cm) }\end{array}$} & \multicolumn{2}{|c|}{ Leaf area $\left(\mathrm{cm}^{2}\right)$} & \multirow{3}{*}{$\begin{array}{l}\text { Days to } \\
\text { flowering }\end{array}$} & \multicolumn{3}{|c|}{ Grain yield $\left(\mathrm{g} / \mathrm{m}^{2}\right)$} & \multicolumn{3}{|l|}{ LER } \\
\hline & & & & \multirow[t]{2}{*}{ Sole } & \multirow[t]{2}{*}{ Intercrop } & & \multicolumn{2}{|c|}{ Sorghum } & \multirow[t]{2}{*}{ Cowpea } & \multirow[t]{2}{*}{ Sorghum } & \multirow[t]{2}{*}{ Cowpea } & \multirow[t]{2}{*}{ Total } \\
\hline & & & & & & & Sole & Inter & & & & \\
\hline \multirow[t]{28}{*}{1981} & Sister lines & & & & & & & & & & & \\
\hline & S 972 & 120 & 63 & 2332 & 2649 & 72 & 52.3 & 37.0 & 22.9 & 0.71 & 0.52 & 1.23 \\
\hline & s 981 & 170 & 73 & 2681 & 2972 & 75 & 72.3 & 55.0 & 21.6 & 0.76 & 0.48 & 1.24 \\
\hline & S 988 & 120 & 72 & 2459 & 3160 & 76 & 38.5 & 26.1 & 18.4 & 0.68 & 0.41 & 1.09 \\
\hline & S 993 & 220 & 90 & 1678 & 2195 & 73 & 26.6 & 25.1 & 22.9 & 0.94 & 0.52 & 1.46 \\
\hline & S 999 & 110 & 73 & 2470 & 3224 & 72 & 43.6 & 41.0 & 23.1 & 0.94 & 0.52 & 1.46 \\
\hline & S 1001 & 130 & 75 & 1818 & 2707 & 76 & 33.3 & 33.9 & 18.5 & 1.02 & 0.44 & 1.46 \\
\hline & S 1003 & 190 & 68 & 2268 & 2794 & 73 & 65.8 & 44.9 & 23.4 & 0.68 & 0.53 & 1.21 \\
\hline & S 1006 & 150 & 68 & 2798 & 3351 & 76 & 34.8 & 34.8 & 20.5 & 1.00 & 0.46 & 1.46 \\
\hline & S 1008 & 140 & 67 & 2266 & 2854 & 72 & 57.5 & 42.3 & 22.4 & 0.73 & 0.50 & 1.23 \\
\hline & S 1018 & 170 & 82 & 2569 & 3344 & 76 & 91.0 & 60.2 & 24.5 & 0.66 & 0.63 & 1.21 \\
\hline & S 1021 & 80 & 47 & 2160 & 1898 & 73 & 47.8 & 23.9 & 28.0 & 0.50 & 0.63 & 1.13 \\
\hline & S 1024 & 110 & 70 & 1961 & 2449 & 72 & 79.7 & 54.9 & 23.0 & 0.69 & 0.52 & 1.21 \\
\hline & Improved lines & & & & & & & & & & & \\
\hline & GP 148 & 100 & 72 & 2750 & 3151 & 73 & 47.8 & 36.3 & 19.5 & 0.76 & 0.44 & 1.20 \\
\hline & Cs 3541 & 130 & 67 & 1927 & 2330 & 72 & 95.0 & 68.5 & 23.5 & 0.62 & 0.53 & 1.15 \\
\hline & $2219 \mathrm{~B}$ & 80 & 53 & 2736 & 3113 & 62 & 86.8 & 34.3 & 29.9 & 0.40 & 0.67 & 1.07 \\
\hline & SPV 351 & 160 & 67 & 2721 & 3413 & 69 & 113.4 & 79.6 & 19.0 & 0.70 & 0.43 & 1.13 \\
\hline & S 902 & 160 & 58 & 2309 & 2949 & 69 & 71.9 & 41.3 & 25.6 & 0.57 & 0.58 & 1.15 \\
\hline & M 66433 & 90 & 60 & 1707 & 1880 & 59 & 11.2 .6 & 50.8 & 33.0 & 0.45 & 0.74 & 1.19 \\
\hline & Hybrids & & & & & & & & & & & \\
\hline & CSH 5 & 170 & 80 & 2732 & 2940 & 69 & 120.4 & 85.9 & 18.3 & 0.71 & 0.41 & 1.12 \\
\hline & CSH 6 & 150 & 65 & 2257 & 2404 & 59 & 172.3 & 105.1 & 24.3 & 0.61 & 0.55 & 1.16 \\
\hline & CSH 9 & 170 & 78 & 1629 & 1521 & 72 & 111.4 & 85.6 & 17.1 & 0.77 & 0.39 & 1.16 \\
\hline & Land races & & & & & & & & & & & \\
\hline & IS 9742 & 220 & 98 & 1473 & 1418 & 59 & 58.7 & 46.5 & 21.5 & 0.79 & 0.48 & 1.27 \\
\hline & E $35-1$ & 250 & 95 & 3222 & 3916 & 82 & 10.4 & 11.7 & 18.2 & 1.12 & 0.41 & 1.53 \\
\hline & Sole cowpea & - & - & - & - & - & - & - & 44.4 & - & - & - \\
\hline \multirow[t]{5}{*}{1982} & Sister lines & & & & & & & & & & & \\
\hline & S993 & 220 & 80 & 2231 & 2567 & 63 & 119.9 & 67.8 & 14.3 & 0.57 & 0.55 & 1.12 \\
\hline & S 999 & 170 & 80 & 2834 & 3086 & 63 & 130.0 & 75.9 & 13.5 & 0.58 & 0.52 & 1.10 \\
\hline & S 1006 & 153 & 85 & 2995 & 3589 & 69 & 125.6 & 78.6 & 13.3 & 0.63 & 0.51 & 1.14 \\
\hline & S 1021 & 90 & 67 & 2586 & 2909 & 62 & 157.4 & 78.6 & 16.2 & 0.50 & 0.61 & 1.11 \\
\hline
\end{tabular}


Improved lines

CHS 5

\section{TABLE 19}

Correlation coefficients between sorghum and cowpea characters in 1981 (above) and 1982 (below) with 21 and 12 DF, respectively

\begin{tabular}{|c|c|c|c|c|c|c|c|c|c|c|c|c|c|c|c|c|c|}
\hline $\begin{array}{l}\text { Sorghum } \\
\text { grain }\end{array}$ & 0.56 & $* *$ & 0.69 & $* * *$ & 0.15 & ns & 0.31 & ns & 0.60 & $* *$ & -0.59 & $* *$ & -0.29 & ns & & & \\
\hline LER & 0.38 & $\mathrm{~ns}$ & 0.52 & $*$ & 0.34 & ns & 0.40 & ns & 0.42 & ns & 0.05 & $\mathrm{~ns}$ & 0.41 & ns & & & \\
\hline $\begin{array}{l}\text { Cowpea } \\
\text { grain }\end{array}$ & -0.45 & $*$ & -0.54 & $* *$ & -0.20 & ns & -0.23 & $\mathrm{~ns}$ & -0.48 & $*$ & 0.24 & ns & -0.09 & ns & -0.68 & $* * *$ & \\
\hline LER & -0.45 & ns & -0.85 & $* * *$ & -0.55 & $*$ & -0.55 & $*$ & -0.74 & $* *$ & 0.03 & $\mathrm{~ns}$ & -0.17 & $\mathrm{~ns}$ & -0.56 & $*$ & \\
\hline \multirow[t]{2}{*}{ LER } & $\begin{array}{r}0.43 \\
-0.30\end{array}$ & $\begin{array}{l}* \\
\mathrm{~ns}\end{array}$ & $\begin{array}{r}0.53 \\
-0.69\end{array}$ & $\begin{array}{l}* * \\
* *\end{array}$ & $\begin{array}{r}0.05 \\
-0.45\end{array}$ & $\begin{array}{l}\text { ns } \\
\text { ns }\end{array}$ & $\begin{array}{r}0.23 \\
-0.41\end{array}$ & $\begin{array}{l}\mathrm{ns} \\
\mathrm{ns}\end{array}$ & $\begin{array}{r}0.45 \\
-0.63\end{array}$ & $\begin{array}{l}* \\
*\end{array}$ & $\begin{array}{r}-0.63 \\
0.07\end{array}$ & $\begin{array}{l}* * \\
\mathrm{~ns}\end{array}$ & $\begin{array}{r}-0.45 \\
0.06\end{array}$ & $\begin{array}{l}* \\
\mathrm{~ns}\end{array}$ & $\begin{array}{r}0.88 \\
-0.03\end{array}$ & $\begin{array}{l}* * * * \\
\mathrm{~ns}\end{array}$ & $\begin{array}{rl}-0.26 & \mathrm{~ns} \\
0.85 & * * *\end{array}$ \\
\hline & $\begin{array}{l}\text { Canopy } \\
\text { height }\end{array}$ & & $\begin{array}{l}\text { Canops } \\
\text { width }\end{array}$ & & $\begin{array}{l}\text { Leaf ar } \\
\text { (sole) }\end{array}$ & & $\begin{array}{l}\text { Leaf ar } \\
\text { (inter) }\end{array}$ & & $\begin{array}{l}\text { Days o } \\
\text { floweri }\end{array}$ & & $\begin{array}{l}\text { Grain: } \\
\text { (sole) }\end{array}$ & yield & $\begin{array}{l}\text { Grain y } \\
\text { (inter) }\end{array}$ & yield & $\begin{array}{l}\text { Sorghu } \\
\text { grain L }\end{array}$ & um & $\begin{array}{l}\text { Cowpea } \\
\text { grain LER }\end{array}$ \\
\hline
\end{tabular}


component crops are not competing for entirely the same environmental resources, and this is probably the case here.

The correlations of sorghum, cowpea and total LERs with each other and with other sorghum characters are presented in Table 19. The salient feature of this correlation matrix is that total LER depends almost entirely on sorghum LER in 1981 and on cowpea LER in the drought year of 1982. Thus characters which contribute to sorghum yield but depress cowpea, such as canopy height, canopy width and days to flowering are positively correlated with total LER in 1981, and negatively or not at all in 1982. This indicates a much greater genotype $X$ environment interaction than is apparent from any single character.

\section{CONCLUSIONS}

The value of a sorghum genotype in intercrop is roughly indicated by its total LER, although of course a genotype which achieved a high LER merely by being low yielding in sole crop would not be valuable. However, as the correlation matrix in Table 19 shows, the sorghum plant characters which produce a high total LER vary widely between seasons. The sorghum genotypes selected will therefore represent a compromise, and this is to be expected since the purpose of intercropping is largely to eliminate risk through compensation by the components. The preceding analyses provide a more detailed understanding of the factors which influence the outcome, and this should allow a more accurate compromise to be reached.

In both sole and intercrop, the grain yield and yield components of sorghum genotypes are largely related to the method by which those genotypes have been bred. However, the intercrop/sole crop ratios are more influenced by whether a genotype is dwarf, and this also has a large effect on cowpea grain yield and pods/plant. The dwarf genotypes are probably too extreme to represent a good compromise for intercropping.

In general, sorghum yield in intercrop is positively associated with characters related to light interception, whereas cowpea yield is negatively associated with such characters. Variables measured in sole crop largely explain sorghum or cowpea yield variation in intercrop, indicating that preliminary selection could be carried out in sole crop. However even when only one component crop is considered, variables measured in intercrop explain yield variation more fully. The correlations between total LER and sorghum plant characters are weak and variable, and evaluation in intercrop will certainly be needed to determine where the best compromise lies on average over a range of environmental conditions

Some idea can, however, be obtained by inspecting the results from specific genotypes in these experiments. The tall wide sorghum genotypes such as S993 and E35-1 gave high LERs in 1981 when soil moisture was not limiting. Thus in this season the sorghum LER varied greatly between 
genotypes and dominated the total LER. In 1982, the tall genotypes which are also late-maturing suffered moisture stress during the reproductive phase after canopy development was complete, and their LERs were reduced. Thus the sorghum LER varied much less than the effect of canopy characters on the cowpea, and cowpea LER dominated the total LER. However, the types of intermediate height such as CS 3541 and CSH 6 , which matured earlier and escaped the moisture stress, gave more stable component LERs and good yields in both seasons. Such genotypes should be selected and, within this category, genotypes having wide canopies should be avoided so that a substantial cowpea yield, which could be particularly important during drought years, can be achieved. Selection for canopy width, which must be conducted at low density, could perhaps begin in the $\mathrm{F}_{2}$ generation.

\section{REFERENCES}

Aiyer, A.K.Y.N., 1949. Mixed cropping in India. Ind. J. Agric. Sci., 19: 439-543.

Arnon, I., 1972. Crop Production in Dry Regions. Volume 2. Leonard Hill, London, England, 683 pp.

Baker, E.F.I., 1974. Research on mixed cropping with cereals in Nigerian farming systems. A system for improvement. In: Proceedings of the International Workshop on Farming Systems, 18-21 November, ICRISAT, Hyderabad, India, pp. 297-301.

Baker, E.F.I. and Yusuf, Y., 1976. Mixed cropping research at the Institute for Agricultural Research, Samaru, Nigeria. In: Proceedings of the Symposium on Intercropping in Semi-Arid Areas, 10-12 May, Morogoro, Tanzania, pp. 17-18.

Clements, F.E., Weaver, J.E. and Hanson, H., 1929. Plant competition. Carnegie Institute of Washington, Publication no. 398.

De Wit, C.T., 1960. On competition. Verslag Landbouwkundige Onderzoek 66.8: 1-82

Draper, N.R. and Smith, H., 1981. Applied Regression Analysis. John Wiley and Sons, Inc., New York, 709 pp.

Finlay, R.C., 1974. Intercropping research and the small famer in Tanzania. In: Proceedings of the Field Staff Symposium IDRC. IDRC, Ottawa, Canada.

Fisher, N.M., 1976. Experiments with maize-bean and maize-potato mixed crops in an area with two short seasons in the highlands of Kenya. In: Proceedings of the Symposium on intercropping in Semi-Arid Areas 10-12 May, Morogoro, Tanzania, pp. $37-38$.

Francis, C.A., Prager, M. and Laing, D.R., 1978a. Genotype by system interactions in climbing bean varieties planted in monoculture and associated with maize. Crop. Sci., 18 : 242-246.

Francis, C.A., Prager, M., Laing, D.R. and Flor, C.A., 1978b. Genotypes $\times$ environment interactions in bush bean cultivars in monoculture and associated with maize. Crop Sci., 18: 237-242.

Galwey, N.W. and Evans, A.M., 1982. Alternative methods of interpreting measurements of resistance to the leafhopper Empoasca kraemiri Ross and Moore in the common bean, Phaseolus vulgaris L. Euphytica, 31 : $225-236$.

Gardner, T.R. and Cracker, L.E., 1981. Bean growth and light interception in a bean maize intercrop. Field Crops Res., 4: 313-320.

Green, J.M., Sharma, D., Reddy, L.J., Saxena, K.B., Gupta, S.C., Reddy, K.C.B.V.S. and Rao, M.R., 1981. Methodology and progress in the ICRISAT pigeonpea breeding 
programme. In: Proceedings of the International Workshop on Pigeonpeas, Vol. 1, 15-19 December 1980, ICRISAT, Hyderabad, India, pp. 437-449.

Hamblin, J., Rowell, J.G. and Redden, R., 1976. Selection for mixed cropping. Euphytica, 25: 97-106.

IRRI, 1974. Cropping systems. In: Annual Report 1974, Los Banos, Phillippines, pp. $323-347$

Krantz, B.A., Virmani, S.M., Singh, S. and Rao, M.R., 1976. Intercropping for increased and more stable agricultural production in the semi-arid tropics. In: Proceedings of the Symposium on Intercropping in Semi-Arid Areas, Morogoro, Tanzania, 10-12 May.

Marshall, B. and Willey, R.W., 1983. Radiation interception and growth in an intercrop of pearl millet/groundnut. Field Crops Res., 7: 141-160.

Mead, R. and Riley, J., 1981. A review of statistical ideas relevant to intercropping research. J. R. Stat. Soc. A, 144: 462-509.

Osiru, D.S.O. and Willey, R.W., 1972. Studies on mixtures of dwarf sorghum and beans (Phaseolus vulgaris L.) with particular reference to plant population. J. Agric. Sci., Camb., 79: 531-540.

Papadakis, J.S., 1941. Small grains and winter legumes grown mixed for grain production. Agron. J., 33: 504-541.

Rao, M.R. and Willey, R.W., 1980. Evaluation of yield stability in intercropping studies on sorghum/pigeonpea. Exp. Agric., 16: 105-116.

Singh, S.R. and Allen, D.J., 1979. Cow pea pests and diseases. Manual Series no. 2, IITA.

Singh, S. and Krantz, B.A., 1976. A brief description of the soils of ICRISAT. ICRISAT, Hyderabad, India.

Stoop, W.A., 1981. Cereal-based intercropping systems for the West African Semi-Arid Tropics, particularly Upper Volta. In: Proceedings of the International Workshop on Intercropping, 10-13 January 1979, Hyderabad, India, pp. 61-68.

Trenbath, B.R., 1976. Plant interactions in mixed communities. In: Proceedings of Multiple Cropping Symposium, American Society of Agronomy, Annual Meeting, 24-29 August, Knoxville, TN, pp. 129-169.

Willey, R.W., 1979. Intercropping - its importance and research needs. Part 1. Competition and yield advantages. Field Crop Abstr., 32:1-10.

Willey, R.W. and Heath, S.B., 1969. The quatitative relationship between plant population and crop yield. Adv. Agron., 21: 281-321.

Willey, R.W. and Osiru, D.S.O., 1972. Studies on mixtures of maize and beans (Phaseolus vulgaris L.) with particular reference to plant population. J. Agric. Sci., Camb., 79: $519-529$.

Williams, B.A. and Austin, R.B., 1977. An instrument for measuring the transmission of short wave radiation by crop canopies. J. Appl. Ecol., 14: 987-991. 